\title{
Low-frequency interlayer vibration modes in two-dimensional layered materials
}

\author{
Jianting Ji, ${ }^{\dagger}$ Shan Dong, ${ }^{\dagger}$ Anmin Zhang, Qingming Zhang ${ }^{*}$ \\ Department of Physics, Beijing Key Laboratory of Opto-electronic Functional Materials \& \\ Micro-nano Devices, Renmin University of China, Beijing 100872, P. R. China
}

\begin{abstract}
Two-dimensional (2D) layered materials have been attracted tremendous research interest because of their novel photoelectric properties. If a single atomic layer instead of individual atoms is taken as a rigid motion object, two unique interlayer vibrations, i.e. compression/breathing and shear motions, at ultra-low frequencies can be expected and actually have been observed in many layered materials. The vibrations stem from the interlayer van der Waals interaction and can be well described by a conventional linear-chain model in most cases. The vibration frequencies strongly depend on layer thickness, which enables an accurate determination of layer numbers. A quick and nondestructive determination of flake thickness is particularly important for the materials, since the physical properties can be dramatically changed in the cases of several atomic layers. As a measure of interlayer coupling, the low-frequency modes are also sensitive to the stacking methods of atomic layers and the overlapping of different kinds of $2 \mathrm{D}$ materials. This allows the modes to play a key role in the applications like van der Waals heterojunctions. In this paper, we will give a brief review on the experimental observations and theoretical understanding of the interlayer modes in several typical 2D systems, as well as their actual and potential applications.
\end{abstract}

Keywords: Layered materials, Raman scattering, interlayer coupling, low-frequency interlayer mode, linear chain model

\footnotetext{
$\dagger$ Contributed equally to this work

* Corresponding author. E-mail address: qmzhang@ruc.edu.cn (Qingming Zhang)
} 


\section{Contents}

\section{Introduction}

1.1 Several typical 2D layered systems

1.2 Simple classification of low-frequency interlayer modes

1.3 Linear chain model

II. Layer-number dependence of low-frequency interlayer modes

2.1 Graphene

2.2 Transition metal dichalcogenides

2.3 Black phosphorus

2.4 Other layered systems

III. Effect of stacking method on low-frequency modes

IV. Low-frequency modes in twisted multilayer materials

V. Summary and outlook 


\section{Introduction}

\subsection{Several typical 2D layered systems}

The successive discovery of fullerene C60 and carbon nanotubes [1,2], stimulated great interest to explore the possibility of two-dimensional carbon materials. It had been predicted that 2D material does not exist in nature due to its thermal instability [3]. Until 2004, the emergence of graphene invalidated the theoretical prediction and opened the door to two-dimensional materials [4]. The sp2 hybridization and covalent in-plane $\sigma$-bonding between neighboring carbon atoms in graphene (Fig. 1a and b), provide a mechanical strength stronger than that of steel by two orders of magnitude [5-7]. Graphene only absorbs $2.3 \%$ of the incident light intensities [8,9]. It has a very high Young's modulus [10] and a thermal conductivity as high as $5300 \mathrm{~W} / \mathrm{m} \cdot \mathrm{K}$ [11]. Its electron mobility at room temperature reaches up to $200,000 \mathrm{~cm}^{2} / \mathrm{V} \cdot \mathrm{s}$ [12]. These outstanding properties make graphene the most promising semiconducting material after silicon. There are two principal phonon modes in graphene, named as $\mathrm{G}$ and $2 \mathrm{D}$ bands. $\mathrm{G}$ band at $\sim 1580 \mathrm{~cm}^{-1}$ originates from in-plane vibrations of sp2-hybridized carbon atoms and has the symmetry of $E_{2 g} .2 D$ band at $2650 \mathrm{~cm}^{-1}$ has an almost doubled frequency of $\mathrm{D}$ band, which is a second-order resonance process and identified as an inter-valley scattering assisted by optical phonons and defects [13]. Therefore, 2D band is related to defects while G band is not. The lineshapes and widths of $\mathrm{G}$ and 2D bands depend on flake thickness, which makes the two modes as an indicator of layer number and flake quality.

Besides graphene, many other materials such as the members of graphene family (h-BN) [14], transition metal sulfides [15], transition metal oxides [16], topological insulators and some mixed phases, etc., have been prepared in 2D form [17-22]. Transition metal dichalcogenides (TMDs) refer to one hexagonal $\mathrm{M}$ metal atomic layer sandwiched between two hexagonal $\mathrm{X}$ chalcogenide atomic layers $\left(\mathrm{MoS}_{2}\right.$, for example shown in Fig. 1c and d). More than 40 graphene-like TMD layered materials can be formed through the combination of $\mathrm{X}(=\mathrm{S}, \mathrm{Se}$ and $\mathrm{Te})$ and $\mathrm{M}(=\mathrm{Mo}, \mathrm{W}, \mathrm{Nb}$, $\mathrm{Re}, \mathrm{Ni}$ and $\mathrm{V}$ ) elements. For the 2D layered materials, a common feature is the 
coexistence of strong in-plane M-X-M covalent bonding and weak interlayer van der Waals force interaction (Fig. 1c and d). There are four Raman-active modes in bulk $\mathrm{MoS}_{2} . \mathrm{E}_{2 \mathrm{~g}}{ }^{1}$ mode at $385 \mathrm{~cm}^{-1}$ and $\mathrm{A}_{1 \mathrm{~g}}$ mode at $410 \mathrm{~cm}^{-1}$ correspond to the in-plane vibrations of Mo-S bond and the out-of-plane vibrations of Mo atoms with S atoms fixed, respectively. In few-layer cases, the frequencies of the two modes exhibit an opposite evolution with flake thickness. The frequency difference between the two modes is $\sim 19 \mathrm{~cm}^{-1}$ in monolayer case and gradually becomes indistinguishable in five or more layer cases. This is attributed to the increasing dielectric screening and the modifications of intralayer and interlayer coupling [23]. The other TMD compounds have the similar behaviors/properties. Atomically thin TMD flakes have many outstanding advantages, like the very high on/off current ratio up to $10^{6}$ [22]. But the relatively low mobility $\left(\sim 200 \mathrm{~cm}^{2} / \mathrm{V} \cdot \mathrm{s}\right)$ limits its industrial applications at present [24].

Recently layered black phosphorus (BP) has invoked enormous interest. Like other 2D materials, black phosphorus has a folded hexagonal honeycomb structure (as shown in Fig. 1e and f), with in-plane covalent bonding of phosphorus atoms and weak interlayer van der Waals force [25-27]. The main Raman modes in bulk BP are $\mathrm{A}_{\mathrm{g}}{ }^{1}, \mathrm{~A}_{\mathrm{g}}{ }^{2}$ and $\mathrm{B}_{2 \mathrm{~g}}[28] . \mathrm{A}_{\mathrm{g}}{ }^{1}$ mode at $\sim 366 \mathrm{~cm}^{-1}$ refers to the out-of-plane vibrations of sulfur atoms while $\mathrm{A}_{\mathrm{g}}^{2}\left(\sim 440 \mathrm{~cm}^{-1}\right)$ and $\mathrm{B}_{2 \mathrm{~g}}\left(\sim 466 \mathrm{~cm}^{-1}\right)$ modes are the in-plane vibrations of sulfur atoms. $\mathrm{A}_{\mathrm{g}}{ }^{1}$ and $\mathrm{B}_{2 \mathrm{~g}}$ modes have little change in peak position with decreasing thickness, but the frequencies of $\mathrm{A}_{\mathrm{g}}{ }^{2}$ mode clearly depend on layer number. $\mathrm{BP}$ has an adjustable direct band gap ranging from $0.33 \mathrm{eV}$ in bulk form to $2.0 \mathrm{eV}$ in monolayer case [29-32]. BP exhibits a large anisotropy in Young's modulus [33,34], Poisson's ratio [35], electron mobility [36], thermal conductivity [37] etc., strongly depending on zigzag or armchair direction [38]. And its mobility in a $\sim 10 \mathrm{~nm}$ flake can reach $1000 \mathrm{~cm}^{2} / \mathrm{V} \cdot \mathrm{s}$ at room temperature [39], which seems promising in semiconducting applications, despite its easy degrade in air[40-44].

\subsection{Simple classification of low-frequency interlayer modes}

Raman scattering is an effective and nondestructive technique to probe electronic properties and lattice vibrations, and has been widely used in the research on 
low-dimensional materials [45]. Through monitoring the subtle change of lattice vibrations, Raman scattering can reflect the influence from the degrees of freedom of charge, spin and lattice [46]. Here we take graphene as an example to demonstrate symmetry considerations of interlayer modes. Bulk graphite has a hexagonal lattice structure with $\mathrm{D}_{6 \mathrm{~h}}{ }^{4}$ symmetry. There are four atoms in each primitive cell, which gives 12 vibration modes at $\Gamma$ points, i.e., $2 \mathrm{E}_{1 \mathrm{u}}+2 \mathrm{~A}_{2 \mathrm{u}}+2 \mathrm{E}_{2 \mathrm{~g}}+2 \mathrm{~B}_{2 \mathrm{~g}}[47,48]$. Among the modes, two $\mathrm{E}_{2 \mathrm{~g}}$ modes are Raman-active and correspond to in-plane optical mode and the rigid-layer shear mode (in-plane relative vibration parallel to layers) respectively. The two $B_{2 g}$ modes are Raman-inactive and correspond to the out-of-plane optical mode and the rigid-layer compression/breathing mode (out-of-plane relative vibration perpendicular to layers) [47]. The symmetry changes with the reduction of flake thickness. The point group with even/odd number of layers is $D_{3 d} / D_{3 h}$, and the compression and shear modes are Raman-active in both cases [49,50]. The rigid-layer compression mode $\left(\mathrm{C}_{\mathrm{N}}\right.$ or $\left.\mathrm{LBM}\right)$ and shear mode $\left(\mathrm{S}_{\mathrm{N}}\right)$ is used to be called low-frequency modes due to their low vibration frequencies $\left(<100 \mathrm{~cm}^{-1}\right)[51]$.

The shear mode $E_{\mathrm{g}}$ locates at $44 \mathrm{~cm}^{-1}$ in graphite, and moves to $42.7 \mathrm{~cm}^{-1}$ in 11-layer graphene, further to $31 \mathrm{~cm}^{-1}$ in bi-layer graphene [52]. Therefore, layer number can be accurately determined by shear mode, particularly at few-layer case. By contrast, the frequency changes of $\mathrm{G}$ and $2 \mathrm{D}$ peaks are very small with layer thickness [52,53]. Similarly, high-frequency $\mathrm{Ag}^{2}$ mode in black phosphorus indicates a change of only $3 \mathrm{~cm}^{-1}$ from monolayer to bulk, while its low-frequency mode has a variation of $19 \mathrm{~cm}^{-1}[28,54,55]$. So the low-frequency mode can be taken as a precise tool for determining the thickness of 2D materials. Furthermore, the shear and compression modes are a good measure of interlayer coupling, and the shear and compression moduluses can be extracted by fitting the experimental data $[51,54,56]$. In addition, the interlayer modes are very sensitive to the stacking styles of atomic layers, the interface and the rotation angle [57-60]. This may be very helpful to the fabrication and characterization of van der Waals heterojunction. So the study of low-frequency modes has become one of the most fundamental issues in 2D layered materials in recent years. 


\subsection{Linear chain model:}

For layered materials, intra-layer covalent bonding is much stronger than the interlayer van der Waals interaction. The low-frequency shear and compression modes arise from the relative vibrations between layers. By taking a rigid atomic layer as one "atom" with large mass, a layered system can be simplified to a one-dimensional monatomic chain with free ends, and only the interaction between the nearest neighboring layers is considered in this chain model. The linear chain model has been well examined in the layered materials grown by epitaxial method [61]. For N-layer materials, totally there are 3(N-1) rigid-plane optical modes in the center of Brillouin zone, including N-1 compression modes along the c axis and N-1 degenerate shear modes. The characteristic displacement vector of the phonon modes are as follows:

$$
\mu_{\mathrm{j}}^{i} \propto \cos \left[\frac{(i-1)(2 j-1) \pi}{2 N}\right]
$$

where $\mathrm{i}$ is the mode index $(\mathrm{i}=2, \ldots, N)$, and $\mathrm{j}$ is the location index of each atom. So the frequency of the i-th vibration mode is obtained:

$$
\omega_{i}^{2}=\frac{\alpha}{2 \pi^{2} c^{2} \mu}\left\{1-\cos \left[\frac{(i-1) \pi}{N}\right]\right\}
$$

where $\mu$ is the mass per unit area of a single atomic layer, $\mathrm{c}$ is the speed of light and $\alpha$ is the interlayer force constant per unit area associated with interlayer coupling. For the modes with the highest frequency $(i=N)$,

$$
\omega_{N}=\sqrt{\left.\frac{\alpha}{2 \pi^{2} c^{2} \mu}\left[1+\cos \frac{(\pi}{N}\right)\right]}
$$

when $N \rightarrow \infty$, it goes to a bulk case, and $\omega_{\infty}=\sqrt{\frac{\alpha}{\pi^{2} c^{2} \mu}}$, which is $\sqrt{2}$ times larger than that of two layers. Formula (2) tells one that there are two opposite trends in the relationship of $\omega$ and $\mathrm{N}$. One is that the frequency increases with increasing layer number, while the other is that the frequency decreases with increasing layer number. Therefore, equation (2) can be resolved to the following form, 
$\cos _{2}=2 \sqrt{1 \pm \cos \left(\frac{n \pi}{N}\right)}$

wherein $\mathrm{n}$ is the mode index, $\mathrm{N} \geqslant 2 \mathrm{n}$ and $\mathrm{n}=1,2,3, \ldots$. . The formula with "+ " applies to the upper branch which frequency increases with number layer, while the formula with "-" applies to the lower branch which has an opposite evolution with layer number. The relation between frequency and layer number based on linear chain model, has been extensively applied to graphene [52,62,63], $\mathrm{MoS}_{2}[23,64-64], \mathrm{MoSe}_{2}$ [59,68], $\mathrm{NbSe}_{2}$ [69], $\mathrm{MoTe}_{2}$ [70] and black phosphorus [54,55,71], etc., and in each case the experimental results are fitted perfectly.

\section{Layer-number dependence of low-frequency interlayer modes}

\subsection{Graphene}

The study on low-frequency modes in graphene was firstly focused on the shear mode $\mathrm{E}_{\mathrm{g}}$ [52]. P.H. Tan et al. made the symmetry analysis and calculated the frequencies of the shear mode by density functional theory (DFT). As shown in Fig. 2a, there are N-1 shear modes for the N-layer sample. As mentioned above, the sample with even/odd layers has a point group $\mathrm{D}_{3 \mathrm{~d}} / \mathrm{D}_{3 \mathrm{~h}}$. Therefore in even-numbered layer the shear mode has the symmetries of $E_{g}$ and $E_{u}$, while in odd layer it is $E^{\prime}$ and E'. One can see that both the highest and the lowest branches are Raman-active. But only the upper branch of the shear mode, which frequency increases with layer number, is visible in graphene, since only the upper branch is symmetry-allowed for the $\mathrm{AB}$ stacking of layered materials (we will discuss this later) (Fig. 2b). Experimentally, it was found that the intensity of the shear mode measured on

mechanically exfoliated graphene with the support of $\mathrm{SiO}_{2} / \mathrm{Si}$ substrate, is very weak (far less than the characteristic G peak), almost invisible in bi-layer case [51]. On the other hand, in the free-standing graphene obtained by drilling on a silicon wafer, the shear mode can be clearly seen. For graphene with 2 to 11 layers and the bulk graphite (Fig. 2b), the uppermost branch of shear mode is clearly visible, and its frequency substantially increases with layer number, while $G$ peak remains almost unchanged. The calculated frequencies are perfectly consistent with the experimental 
values. And the linear chain model provides a perfect fitting curve (Fig. 2c). Equation (3) gives $\omega_{2}=\sqrt{\frac{\alpha}{2 \pi^{2} c^{2} \mu}}$ where the quantity $\alpha$, which is about $12.8 \times 10^{18} \mathrm{Nm}^{-3}$ in this case, can be determined by the $\omega_{2}$ formula. This indicates that the hardening of the shear mode with increasing layer number in the AB stacked graphene, is caused by the increase of overall restoring force rather than the change of interlayer coupling [52]. The shear modulus of $4.3 \mathrm{GPa}$ can be calculated by the use of $\mathrm{C}_{44}=\alpha \mathrm{t}$ (where $\mathrm{t}$ is the interlayer spacing). The value is consistent with the result given by inelastic X-ray scattering [52,72].

It is hard to observe a compression/breathing mode with first-order Raman measurements. The compression mode (LBM or $\mathrm{ZO}^{\prime}$ ) located at about $100 \mathrm{~cm}^{-1}$ has been studied in bulk graphite [62]. C. Lui et al. measured the double overtone 2ZO' of $\mathrm{ZO}^{\prime}$ mode in few-layer graphene utilizing double-resonance Raman technique. As seen in Fig. 3c, there are two conduction bands (c1, c2) and two valence bands (v1, v2) in 2-layer graphene. This provides four possible resonance processes, i.e. P11, P12, P21, P22 for intra-valley double-phonon scattering. Only the two processes P11 and $\mathrm{P} 22$ which correspond to $2 \mathrm{ZO}^{\prime-}$ and $2 \mathrm{ZO}^{\prime+}$, are allowed, since P12, $\mathrm{P} 21$ is restricted in the high-symmetry points of the Brillouin zone center. It can be seen from Fig. 3a that there are two peaks in the vicinity of $180 \mathrm{~cm}^{-1}$ in 2-layer sample. They are similar to the high-order 2D peak. For N-layer sample, there are N-1 branches of compression mode from $2 \mathrm{ZO}_{\mathrm{N}}{ }^{(1)}$ to $2 \mathrm{ZO}_{\mathrm{N}}{ }^{(\mathrm{N}-1)}$. For each compression mode, its frequency increases with increasing layer number and decreases with increasing mode index. The thickness dependence of ZO' mode frequency is given by 2ZO' central peak and can be well described with the theoretical model (Fig. 3b).

\subsection{Transition metal dichalcogenides}

$\mathrm{MoS}_{2}$ has a structure of $\mathrm{AB}$ stacking $\left(\mathrm{D}_{6 \mathrm{~h}}{ }^{4}, \mathrm{P} 63 / \mathrm{mmc}\right)$ in which one Mo atomic layer is sandwiched between two layers of $\mathrm{S}$ atoms [73-76]. Its primitive cell contains six atoms. So it has 18 branches of phonons at $\Gamma$ point, namely $\Gamma_{\text {bulk }}=$ $\mathrm{A}_{1 \mathrm{~g}}+2 \mathrm{~B}_{2 \mathrm{~g}}+\mathrm{E}_{1 \mathrm{~g}}+2 \mathrm{E}_{2 \mathrm{~g}}+2 \mathrm{~A}_{2 \mathrm{u}}+\mathrm{B}_{1 \mathrm{u}}+2 \mathrm{E}_{1 \mathrm{u}}+\mathrm{E}_{2 \mathrm{u}}[66,74-76]$, where $\mathrm{A}_{1 \mathrm{~g}}$ mode at $\sim 408 \mathrm{~cm}^{-1}$ 
and $\mathrm{E}_{2 \mathrm{~g}}{ }^{1}$ mode at $\sim 382 \mathrm{~cm}^{-1}$ correspond to the out-of-plane vibrations of $\mathrm{S}$ atoms and in-plane vibrations of Mo and S atoms, respectively. The frequencies of both modes change with layer number and the difference in frequency between the two modes increases from $19 \mathrm{~cm}^{-1}$ to $26 \mathrm{~cm}^{-1}$ with the thickness variation from monolayer to bulk $[23,77]$. The low-frequency $\mathrm{E}_{2 \mathrm{~g}}{ }^{2}$ and $\mathrm{B}_{2 \mathrm{~g}}{ }^{2}$ modes correspond to the shear mode and compression vibrations, respectively (Fig. $4 \mathrm{a}$ ). $\mathrm{B}_{2 \mathrm{~g}}{ }^{2}$ mode is invisible in bulk since it is a Raman inactive in this case. The point group of few-layer $\mathrm{MoS}_{2}$ is $\mathrm{D}_{3 \mathrm{~d}}$ in even-layer sample and $\mathrm{D}_{3 \mathrm{~h}}$ in odd-layer sample, and the compression mode becomes Raman-active. The frequency difference of shear mode $\mathrm{E}_{\mathrm{g}}$ in bilayer and bulk samples can reach up to $10 \mathrm{~cm}^{-1}$ as shown in Fig. 4b. The compression mode $A_{1 g}$ exhibits an even larger frequency variation $\left(41 \mathrm{~cm}^{-1}\right.$ for bilayers and $12 \mathrm{~cm}^{-1}$ for 7 layers). Its width is significantly reduced with increasing layer number and the peak eventually disappears in bulk sample. Therefore, the layer number of few-layer $\mathrm{MoS}_{2}$ can be accurately determined with the two low-frequency modes. Both modes well follow the simple one-dimensional monoatomic chain model and the experimental data can be fitted perfectly (Fig. 4c and d) using formula (4). The fitting gives the interlayer force constant per unit area $\alpha_{\mathrm{S}}=2.9 \times 10^{19} \mathrm{~N} \mathrm{~m}^{-3}$ and $\alpha_{\mathrm{C}}=8.6 \times 10^{19} \mathrm{~N} \mathrm{~m}^{-3}$ with $\mathrm{C}_{44} \sim$ 17.9 GPa and $\mathrm{C}_{33} \sim 52.0 \mathrm{GPa}[59,66]$. The ratio of the in-plane shear force constant to the interlayer one, can be estimated to be about 100 [56] using a simple relationship $\omega^{2} \sim \mathrm{K} / \mathrm{M}$, where $\mathrm{K}$ is the force constant and $\mathrm{M}$ the atomic mass [56,78]. For the compression force constant, we have approximately the same ratio. Similar to graphene, in principle there are $\mathrm{N}-1$ branches of degenerate shear modes and N-1 branches of compression modes for N-layer $\mathrm{MoS}_{2}$. Besides the shear mode with the highest frequency and the compression mode with the lowest frequency, the interlayer modes for $n=3,5$ are also observed [64]. The fitting difference between the diatomic and monatomic chain models, is almost negligible.

Other TMD compounds with $\mathrm{AB}$ stacking have the similar hexagonal lattice structure as that of $\mathrm{MoS}_{2}$. The in-plane strong M-X-M bonding and the weak van der Waals interlayer coupling coexist in $\mathrm{MX}_{2}$. The interlayer vibration modes have also been observed in other TMD materials such as $\mathrm{MoSe}_{2}, \mathrm{WSe}_{2}, \mathrm{MoTe}_{2}$ and $\mathrm{ReS}_{2}$ at low 
frequencies $[59,66,68,70,79,80]$. The low-frequency mode of $\mathrm{MoSe}_{2}$ under parallel polarization is shown in Fig. 5a, in which no peak is seen in monolayer case but it emerges in the samples with more than two layers. The shear modes have the highest, the third highest and the fifth highest frequencies respectively, which reduce rapidly with increasing layer number. It locates at about $19 \mathrm{~cm}^{-1}$ in bilayer case, slightly less than that of $\mathrm{MoS}_{2}$. Fig. $5 \mathrm{~b}$ indicates the two low-frequency modes in $\mathrm{WSe}_{2}$ under parallel polarization. The compression mode is symmetry-prohibited under cross polarization. The upper branch of the shear mode $\left(\mathrm{S}_{1}, \mathrm{~S}_{2}\right)$ and the lower branch of the compression mode $\left(\mathrm{B}_{1}, \mathrm{~B}_{2}\right)$, are observed under parallel polarization. The two branches have an opposite evolution with layer number, i.e. blue shift for $S_{1}, S_{2}$ and red shift for $\mathrm{B}_{1}, \mathrm{~B}_{2}$. The low-frequency modes in the two TMDs also satisfy linear chain model exactly and the fitting gives $\mathrm{C}_{44}=18.6 \mathrm{GPa}$ and $\mathrm{C}_{33}=52.1 \mathrm{GPa}$ in $\mathrm{WSe}_{2}$. Both values are slightly larger than those of $\mathrm{MoS}_{2}[66]$.

In bulk/bilayer $\mathrm{MoTe}_{2}$, both the shear mode $\left(\mathrm{B}_{2 \mathrm{~g}} / \mathrm{A}_{1 \mathrm{~g}}\right)$ and the compression mode $\left(E_{2 \mathrm{~g}} / \mathrm{E}_{\mathrm{g}}\right)$ are Raman-active [70]. Fig. 5c shows the Stokes and anti-Stokes Raman spectra under $\mathrm{XX}$ and $\mathrm{XY}$ polarization configurations in $\mathrm{N}$-layer $(\mathrm{N}=1$ to 12$)$ and bulk $\mathrm{MoTe}_{2}$. Only $\mathrm{A}_{1 \mathrm{~g}}$ compression mode is visible in the parallel-polarization (XX) channel, while both modes are observed in the cross polarization (XY) channel. And the intensity of compression mode in XX channel is one order of magnitude larger than that in XY channel. The shear (compression) mode exhibits a gradual softening (hardening) with increasing layer number. The evolution well follows linear chain model and hence can be taken as an accurate way of determining layer number, as seen in other layered TMD materials.

$\mathrm{ReS}_{2}$ is a $2 \mathrm{D}$ layered semiconductor with novel electronic properties and the stable phase in monolayer case has a distorted $1 \mathrm{~T}$ structure $[79,80]$. The anisotropy in $\mathrm{ReS}_{2}$ allows two non-degenerate interlayer shear mode $\left(\mathrm{S}_{\|}\right.$and $\left.\mathrm{S}_{\perp}\right)$ and one compression mode, all of which are observed in the Raman spectra below $50 \mathrm{~cm}^{-1}$ (Fig. 5d). Compared to the theoretical calculations, they are assigned to $S_{\|}\left(13 \mathrm{~cm}^{-1}\right)$, $\mathrm{S}_{\perp}\left(16.5 \mathrm{~cm}^{-1}\right)$ and $\mathrm{B}$ modes $\left(28 \mathrm{~cm}^{-1}\right)$, respectively. Actually it is hard to distinguish the shear modes from other low-frequency modes due to their weak intensities (Fig. 
$5 b)$. The compression mode (B) shifts to lower frequencies with increasing thickness. $\mathrm{B}_{1}$ mode locates at $\sim 8 \mathrm{~cm}^{-1}$ in 10-layer case, while it has a large frequency change of $\sim 20 \mathrm{~cm}^{-1}$ from 2- to 10-layer cases. The higher-order compression modes $\left(\mathrm{B}_{2}, \mathrm{~B}_{3}, \mathrm{~B}_{4}\right)$ are also visible in the spectra, and all the observed compression modes can be fit by linear chain model.

\subsection{Black phosphorus}

Black phosphorus has attracted more and more attention recently because of its unique structure and electronic/optical properties. Black phosphorus has an orthogonal structure $\left(\mathrm{D}_{2 \mathrm{~h}}{ }^{18}\right)[81,82]$. One phosphorus atom is covalently bonded with its three nearest neighbors. And it is generally believed that the interlayer coupling also arises from weak van der Waals interaction. There are four atoms in a primitive cell, which allows 12 phonon modes, namely $\Gamma_{\text {bulk }}=2 A_{g}+B_{1 g}+B_{2 g}+2 B_{3 g}+A_{2 u}+2 B_{1 u}+$ $2 \mathrm{~B}_{2 \mathrm{u}}+\mathrm{B}_{3 \mathrm{u}}$, wherein $2 \mathrm{~A}_{\mathrm{g}} 、 \mathrm{~B}_{1 \mathrm{~g}} 、 \mathrm{~B}_{2 \mathrm{~g}}, 2 \mathrm{~B}_{3 \mathrm{~g}}$ modes are Raman-active and refer to in-plane relative vibrations [81]. The $D_{2 h}$ symmetry remain unchanged in few-layer black phosphorus. But different from bulk, the point group is $\mathrm{D}_{2 \mathrm{~h}}{ }^{7}$ (pmna) for odd layers and $\mathrm{D}_{2 \mathrm{~h}}{ }^{11}$ (pbca) for even layers [82]. In few-layer case, the classification of vibration modes is changed to $\Gamma_{n-l a y e r}=n\left(2 A_{g}+B_{1 g}+B_{2 g}+2 B_{3 g}+A_{2 u}+2 B_{1 u}+2 B_{2 u}+B_{3 u}\right)$, where the Raman-active $A_{g}, B_{1 g}$ and $B_{2 g}$ modes are the out-of-plane compression mode and the shear mode along zigzag and armchair directions, respectively [55].

Due to Raman selection rules, only the $A_{g}$ mode can be observed under $\mathrm{z}(\mathrm{xx}) \mathrm{z}$ configuration. Raman measurements on a large number of BP flakes indicate that two branches of low-frequency modes exhibit a significant evolution with layer number (Fig. 6a), while no low-frequency peak is seen in monolayer and bulk samples[54]. It means that the modes appearing at low frequencies are interlayer vibrations. And only the compression mode is allowed according to the symmetry analysis on few-layer black phosphorus. The mode near $80 \mathrm{~cm}^{-1}$ can be well fitted by linear chain model, and is further identified as a conventional interlayer compression/breathing vibration perpendicular to sulphur planes (Fig. $6 \mathrm{~b}$ and $\mathrm{d}$ ). The modes at relatively high frequencies are determined to be the second and fourth compression ones. 
The mode around $30 \mathrm{~cm}^{-1}$ is of particular interest. Its redshift with layer number can be well described by $1 / \sqrt{N}$ rather than $\cos _{2}=\sqrt{1 \pm \cos \left(\frac{n \pi}{(N)}\right)}$ or $1 / \mathrm{N}[54]$. This suggests that the vibration represents a collective compression motion relative to substrate by combing all the layers as a whole rather than a single layer (Fig. 6c and e). This mode is called as collective compression mode (CCM). It means that the interlayer coupling is quite strong to bind the individual layers together. The mode has also been observed in $\mathrm{KBr}$ films epitaxially grown on a $\mathrm{NaCl}$ substrate [83]. The layer-number dependence of the mode frequency is quadratic and its intensity has a weak dependence on the polarization of incident light, which is consistent with the conclusion mentioned above (Fig. $6 \mathrm{f}$ and g). The ratio of intralayer to interlayer force constants can be estimated through the ratio of the intralayer vibration mode $\mathrm{A}_{\mathrm{g}}{ }^{1}$ $\left(\sim 366 \mathrm{~cm}^{-1}\right)$ to the compression mode $\mathrm{Ag}\left(71 \mathrm{~cm}^{-1}\right)$ in bilayer BP. The ratio is about 26, much smaller than that of $\mathrm{MoS}_{2}$ [54]. The ratio of intra- and interlayer force constants are the interlayer binding energy per unit are listed in Table 1, compared to those of $\mathrm{MoS}_{2}$ and graphene. The values in BP are significantly larger than those of the other two samples. This evidences that the interlayer coupling in few-layer BP is different from the case of MoS2 and may paly an important role in understanding the structural and electronic properties of few-layer BP and its applications. The strong interlayer coupling is considered to arise from the electron hybridization of lone pair of electrons besides van der Waals interlayer interaction [84].

The low-frequency modes in multi-layer BP can also be used to effectively determine the flake thickness. The high-frequency modes $\mathrm{A}_{\mathrm{g}}{ }^{1}$ and $\mathrm{B}_{2 \mathrm{~g}}$ have little frequency shift with varying the layer number, and $\mathrm{A}_{\mathrm{g}}{ }^{2}$ mode only has a shift of $\sim 3$ $\mathrm{cm}^{-1}$ from monolayer to five-layer and is not effective for the determination of layer number, particularly in few-layer case. On the other hand, the CCM and interlayer compression mode exhibit a large shift in frequency with flake thickness. From bilayer to bulk, the frequency change of CCM and the compression mode are $32 \mathrm{~cm}^{-1}$ and $19 \mathrm{~cm}^{-1}$ respectively[54]. Furthermore, both modes are still very sensitive to the thickness even in the thicker samples with ten or more layers. So they can provide an 
accurate and effective way to determine the layer number of few-layer BP. This is particularly helpful for the studies of BP, since the easy degradation in air makes it difficult to determine BP flake thickness with conventional techniques like optical imaging.

Table I Comparison of the interlayer coupling in $\mathrm{BP}, \mathrm{MoS}_{2}$ and graphene. Here is the experimental per unit area interlayer force constant $\alpha, \mathrm{K}_{\text {intra }} / \mathrm{K}_{\text {inter }}$ (intralayer/interlayer spring force constant)and interlayer binding energy per unit area $E_{B}$ which is calculated by density functional Optb88-vdW, DFT-D2, and Optb86-vdW.

\begin{tabular}{llllll}
\hline & $\mathbf{a}$ & & \multicolumn{3}{c}{$\mathbf{E}_{\mathbf{B}}\left(\mathbf{m e V} / \AA^{2}\right)$} \\
\cline { 5 - 6 } & $(\boldsymbol{\alpha}$ & $\mathbf{K}_{\text {intra }} / \mathbf{K}_{\text {inter }}$ & Optb88-vdW & DFT-D2 & Optb86-vdW \\
\cline { 5 - 6 } & $\left.\mathbf{1 0}^{\mathbf{1 8}} \mathbf{N} / \mathbf{m}^{\mathbf{3}}\right)$ & & 31 & 23 & 35 \\
\hline black P & 127 & 26 & 26 & 18 & 27 \\
\hline $\mathbf{M o S}_{2}$ & 29 & 100 & 26 & 21 & 26 \\
\hline graphene & 12.8 & $/$ & &
\end{tabular}

\subsection{Other layered systems}

Distinguished from graphene, TMD and BP layered materials discussed above, the V-VI compounds $\mathrm{Bi}_{2} \mathrm{Te}_{3}$ and $\mathrm{Bi}_{2} \mathrm{Se}_{3}$ are well-known topological insulators [85]. The layered $\mathrm{Bi}_{2} \mathrm{X}_{3}(\mathrm{X}=\mathrm{Te}, \mathrm{Se})$ is structured by the tight stacking of hexagonal atomic layers along c axis. Each few-quintuple-layer (QL) is composed of five ABC-stacked atomic layers which are covalently bonded through X-Bi-X-Bi-X chains (Fig. 7c\&d). The neighboring QL layers are coupled through van der Waals interaction. It has a rhombohedral lattice with point group $\mathrm{D}_{3 \mathrm{~d}}{ }^{5}$ (R-3m) in bulk, which gives 15 branches of optical phonon modes, i.e., $\Gamma_{\text {bulk }}=2 \mathrm{~A}_{1 \mathrm{~g}}+2 \mathrm{E}_{\mathrm{g}}+3 \mathrm{~A}_{2 \mathrm{u}}+3 \mathrm{E}_{\mathrm{u}}$ [86]. The symmetry changes to $\mathrm{D}_{3 \mathrm{~d}}{ }^{3}(\mathrm{R}-3 \mathrm{~m} 1)$ with a hexagonal lattice in few-layer cases, and the primitive cell of N-QL system contains $5 \mathrm{~N}$ atoms. The optical modes are $\Gamma_{\mathrm{odd}}=(5 \mathrm{~N}-1) / 2\left(\mathrm{~A}_{1 \mathrm{~g}}+\mathrm{E}_{\mathrm{g}}\right)$ $+(5 \mathrm{~N}+1) / 2\left(\mathrm{~A}_{2 \mathrm{u}}+\mathrm{E}_{\mathrm{u}}\right)$ for odd-QL systems and $\Gamma_{\text {even }}=5 \mathrm{~N} / 2\left(\mathrm{~A}_{1 \mathrm{~g}}+\mathrm{E}_{\mathrm{g}}+\mathrm{A}_{2 \mathrm{u}}+\mathrm{E}_{\mathrm{u}}\right)$ for even-QL systems, where $\mathrm{A}_{1 \mathrm{~g}}$ and $\mathrm{E}_{\mathrm{g}}$ are Raman-active shear and 
compression/breathing modes, respectively. According to the Raman tensors, both $\mathrm{A}_{1 \mathrm{~g}}$ and $\mathrm{E}_{\mathrm{g}}$ modes are visible in parallel polarization configuration while only $\mathrm{E}_{\mathrm{g}}$ mode can be seen in the cross polarization channel.

The low-frequency Raman spectra of few-layer $\mathrm{Bi}_{2} \mathrm{Te}_{3}$ and $\mathrm{Bi}_{2} \mathrm{Se}_{3}$ under $-\mathrm{Z}(\mathrm{XX}) \mathrm{Z}$ backscattering configuration are shown in Fig. 7a\&b. The redshifts of the shear mode (S1) and compression mode (B1) with thickness are observed in both materials, and the frequency evolution still well follows linear chain mode (Fig. 7c). Interestingly, there appears a particular mode in 2/3-layer $\mathrm{Be}_{2} \mathrm{Te}_{3}$, which is out of the classification of the optical modes at $\Gamma$ points and cannot be described simply by linear chain model. The experimental results are in good agreement with the theoretical values of the effect of substrate is taken into account. The acoustic mode changes to optical interface mode (I) in this case (Fig. 7d). It can be understood as the vibration of the whole atomic layers relative to substrate, which is similar to the CCM in multilayer $\mathrm{BP}$ and indicates a relatively strong interlayer coupling.

\section{Effect of stacking method on low-frequency modes}

The usual stacking methods are $\mathrm{ABA}$ and $\mathrm{ABC}$ in $2 \mathrm{D}$ layered materials. They demonstrate considerable differences in electronic structures, manipulation of energy band gap, quantum Hall effect, infrared absorption and other aspects [87-92]. In principle, the impact of stacking on interlayer phonon modes is very small (Fig. 8b), since it stems from the weak van der Waals interlayer coupling. However, the symmetries of $\mathrm{ABA}$ - and $\mathrm{ABC}$-stacking are significantly different [58]. For three-layer graphene, ABA stacking corresponds to point group $\mathrm{D}_{3 \mathrm{~h}}$ with 18 branches of standard phonon modes at $\Gamma$ points, namely $2 \mathrm{~A}_{1}{ }^{\prime}+4 \mathrm{E}^{\prime}+4 \mathrm{~A}_{2}{ }^{\prime}+2 \mathrm{E}^{\prime}$. E' and $\mathrm{E}^{\prime \prime}$ modes are the uppermost and lowest branches of shear mode, and $\mathrm{A}_{1}$ ' is the lowest branch of compression mode. As shown in Fig. 8a, all the three low-frequency branches are Raman active. But for $\mathrm{ABC}$ stacking, the point group becomes $\mathrm{D}_{3 \mathrm{~d}}$ with inversion center. It gives 18 branches of phonon modes at $\Gamma$ points as $3 \mathrm{~A}_{\mathrm{g}}{ }+3 \mathrm{E}_{\mathrm{g}}+3 \mathrm{~A}_{\mathrm{u}}+3 \mathrm{E}_{\mathrm{u}}$. In this case, $\mathrm{E}_{\mathrm{g}}$ and $\mathrm{A}_{\mathrm{g}}^{1}$ modes are the lowest branch of shear mode and the lowest 
branch of compression mode, respectively. And the rest of the modes are infrared-active vibrations. Fig. 8c shows Raman measurements on three-layer ABAand $\mathrm{ABC}$-stacking graphene. The identified compression mode appears at $57 \mathrm{~cm}^{-1}$ in both $\mathrm{ABA}$ - and $\mathrm{ABC}$-stacking graphene. This is because the nearest-neighboring interlayer coupling is the same for $\mathrm{ABA}$ and $\mathrm{ABC}$ stacking and the next nearest neighbor interaction is so small that it can be negligible. However, the effect of stacking on low-frequency modes is clear in a lower frequency region. By comparison with the theoretical values, on can see shows that the uppermost branch of $\mathrm{C}$ appears in ABA stacking graphene while the lowest branch of $\mathrm{C}^{\prime}$ occurs in $\mathrm{ABC}$ stacking graphene. ABA stacking has mirror symmetry and ABC stacking has antisymmetry. That is why they are obviously distinct in Raman activity and phonon intensity [58].

The dependence of low-frequency mode on stacking methods has also been well verified in other 2D materials. For example, the upper branch of shear mode was also observed in the mechanically exfoliated $\mathrm{AB}$ stacking $\mathrm{MoS}_{2}$ and $\mathrm{WSe}_{2}$ [63,64-66], while the lowest shear mode appears in $\mathrm{ABC}$ stacking $\mathrm{Bi}_{2} \mathrm{Se}_{3}$ and $\mathrm{Bi}_{2} \mathrm{Te}_{3}$ [57,86]. Two kinds of stacking methods, i.e. $\mathrm{AB}$ and $\mathrm{ABC}$, are available in the epitaxially grown $\mathrm{MoSe}_{2}$. Therefore, not only the upper branch but also the lower branch has been observed $[59,68]$. It seems that the stacking effect are generally applicable to the interlayer modes of many $2 \mathrm{D}$ systems, since it comes from symmetry rather than materials.

\section{Low-frequency modes in twisted multilayer materials}

The twisted multilayer flakes formed by the overlapping of few-layer ones have many promising applications in electronic devices and optoelectronic fields. The low-frequency modes of such multiplayer systems contain some key information on interlayer coupling. For $m+n$ layers of graphene $[t(m+n) L G, m \neq n]$, the symmetry is $\mathrm{C}_{3}$ with $\Gamma_{\text {Raman }}=\mathrm{A}+\mathrm{E}$, where the compression mode has A symmetry and the shear mode E symmetry. According to the Raman tensors, A and E modes can be observed in parallel polarization, while E mode is prohibited in cross polarization channel [93].

Fig. 9 shows Raman spectra of six twisted graphene samples $t(1+2) L G$, 
$\mathrm{t}(1+1+1) \mathrm{LG}, \mathrm{t}(1+3) \mathrm{LG}, \mathrm{t}(2+2) \mathrm{LG}, \mathrm{t}(2+3) \mathrm{LG}, \mathrm{t}(5+5) \mathrm{L}$. The peak at $\sim 109.9 \mathrm{~cm}^{-1}$ in $\mathrm{t}(1+2) \mathrm{LG}$ and the peak at $108.8 \mathrm{~cm}^{-1}$ in $\mathrm{t}(1+1+1) \mathrm{LG} \mathrm{h}$ are close to the calculated $\operatorname{pos}\left(\mathrm{LBM}_{31}\right)=110.8 \mathrm{~cm}^{-1}$ but quite far away from $\operatorname{pos}\left(\mathrm{LBM}_{21}\right)=90.5 \mathrm{~cm}^{-1}$. The mode is assigned as the compression/breathing vibration of tri-layer graphene. Similarly, in $\mathrm{t}(1+3) \mathrm{LG}$ and $\mathrm{t}(2+2) \mathrm{LG}$ the two compression modes are almost identical and consistent with the calculated values of 4-layer graphene. This suggests that the compression mode in $\mathrm{m}+\mathrm{n}$ layer twisted graphene is little affected by interface distortion. The interlayer compression coupling is almost constant and the frequency only depends on the total number of layers N. On the other hand, it is very different for shear mode. The two peaks $\mathrm{C}_{31}$ and $\mathrm{C}_{32}$ in $\mathrm{t}(1+3) \mathrm{LG}$ and $\mathrm{C}_{21}$ were identified as shear modes. And the three peaks were also found in $\mathrm{t}(2+3) \mathrm{LG}$, while $\mathrm{C}_{51}, \mathrm{C}_{53}$ and $\mathrm{C}_{54}$ are visible in $\mathrm{t}(5+5) \mathrm{LG}$. It indicates that the shear modes in $(\mathrm{m}+\mathrm{n})$-layer graphene seem to come from the separate $\mathrm{mLG}$ and $\mathrm{nLG}$. This is due to the fact that the periodic mismatch between the twisted layers reduces interlayer coupling force and the mode frequency.

The shear mode in Bernal stacking graphene can be observed only in free-standing form on silicon and no sign of compression mode is seen [52]. The low-frequency modes can be observed very easily in the twisted overlapped graphene and their intensities are also much enhanced. In the overlapped multilayer systems made of different kinds of few-layer 2D materials, such as $\mathrm{MoS}_{2} / \mathrm{MoSe}_{2}$ and $\mathrm{MoS}_{2} / \mathrm{WSe}_{2}$, low-frequency modes are also sensitive to the composition of flakes, the contact surface condition and the overlapping angle etc. [60]. The studies of low-frequency modes for overlapped layered materials is a fundamental issue and substantially contributes to the understanding and development of $2 \mathrm{D}$ van der Waals heterojunctions.

\section{Summary and outlook}

The low-frequency modes originating from relative vibrations between layers, are particularly meaningful for the study of 2D layered materials. They can not only accurately determine the layer number of few-layer samples, but also provides a good 
measure of the strength of interlayer interaction. By determining $\alpha$ with mode frequencies, the interlayer shear and compression modulus can be conveniently extracted $[23,54,64-66]$. This offers a simple but effective and non-destructive way compared to other methods of modulus measurement.

A key application of low-frequency modes is to monitor the interlayer coupling of van der Waals heterojunction, in which the intrinsic electronic properties of two individual layers are modified due to the weak interlayer coupling at contact surface. The atomic-layer stacking of different kinds of 2D layered materials, will output new features and applications in optics, electronics and other aspects [94-99]. For instance, the photoluminescence (PL) of $\mathrm{MoSe}_{2}$ is strongly suppressed in $\mathrm{MoSe}_{2} /$ graphene heterojunction, which indicates that there exists a very fast charge transfer of photo-generated carriers between $\mathrm{MoSe}_{2}$ and graphene [98]. And the interlayer coupling can be adjusted by inserting dielectric layer into heterojunction. The PL peaks are changed when monolayer h-BN is inserted in $\mathrm{WSe}_{2} / \mathrm{MoS}_{2}$ heterojunction. If the heterojunction contains tri-layer h-BN, the PL peaks seems consistent with those of monolayer $\mathrm{MoS}_{2}$ and monolayer $\mathrm{WSe}_{2}$, suggesting that the interlayer coupling between two different atomic layers can be ignored in this case. PL peaks of $\mathrm{WSe}_{2} / \mathrm{MoS}_{2}$ heterojunction without dielectric layer are weaker than those of monolayer $\mathrm{WSe}_{2}$ and $\mathrm{MoS}_{2}$, due to the strong coupling of interlayer charge carriers. Because the direct absorption and indirect emission exists in heterojunction, about $100 \mathrm{mev}$ of stokes-like shift appears in PL peaks and the lowest energy absorption peaks [99]. In addition, there are many unique properties of van der Waals heterojunction in spintronics, excitons etc. [100-102]. The frequencies of low-frequency modes reflect not only the basic information on thickness or layer number of the heterostructure but also some key factors like coupling strength and condition of its contact surface. This suggests low frequency modes may play a key role in the research of van der Waals heterojunction. 


\section{Acknowledgments}

This work was supported by the Ministry of Science and Technology of China (973 projects: 2012CB921701) and the NSF of China. Q.M.Z. was supported by the Fundamental Research Funds for the Central Universities, and the Research Funds of Renmin University of China (10XNI038).

\section{References}

[1] H. W. Kroto, J. R. Heath, S. C. Obrien, R. F. Curl, R. E. Smalley, Nature 318 (1985) 162.

[2] S. Iijima, Nature 354 (1991) 56.

[3] L. Sparks, G. V. Hoven, Solar Phys. 97 (1985) 283.

[4] K. S. Novoselov, A. K. Geim, S. V. Morozov, D. Jiang, Y. Zhang, S. V. Dubonos, I. V. Grigorieva, A. A. Firsov, Science 306 (2004) 666.

[5] T. Ohta, A. Bostwick, T. Seyller, K. Horn, E. Rotenberg, Science 313 (2006) 951.

[6] T Enoki, M Endo, M Suzuki, Graphite intercalation compounds and applications Oxford University Press (2003).

[7] M. J. Allen, V. C. Tung, R. B. Kaner, Chem. Rev. 110 (2010) 132.

[8] R. R. Nair, P. Blake, A. N. Grigorenko, K. S. Novoselov, T. J. Booth, T. Stauber, N. M. R. Peres, A. K. Geim, Science 320 (2008) 1308.

[9] P. Blake, P. D. Brimicombe, R. R. Nair, T. J. Booth, D. Jiang, F. Schedin, L. A. Ponomarenko, S. V. Morozov, H. F. Gleeson, E. W. Hill, K. Andre, G. Kostya, S. Novoselov, Nano Lett. 8 (2008) 1704.

[10] F. Liu, P. Ming, J. Li, Phys. Rev. B 76 (2007) 064120.

[11] A. A. Balandin, Nat. Mater. 10 (2011) 569.

[12] K. I. Bolotin, K. J. Sikes, Z. Jiang, M. Klima, G. Fudenberg, J. Hone, P. Kim, H. L. Stormer, Solid State Commun. 146 (2008) 351.

[13] A. C. Ferrari and D. M. Basko, Nat. Nanotechnol. 8 (2013) 235.

[14] C. R. Dean, A. F. Young, I. Meric, C. Lee, L. Wang, S. Sorgenfrei, K. Watanabe, T. Taniguchi, P. Kim, K. L. Shepard, J. Hone, Nat. Nanotechnol. 5 (2010) 722.

[15] J. A. Wilson, A. D. Yoffe, Adv. Phys. 18 (1969) 193.

[16] M. Osada, T. Sasaki, Adv. Mater. 24 (2012) 210.

[17] A. Gupta, T. Sakthivel, S. Seal, Prog. Mater. Sci. 73 (2015) 44.

[18] A. B. Kaul, J. Mater. Res. 29 (2014) 348.

[19] X. Song, J. Hu, H. Zeng, J. Mater. Chem. C 1 (2013) 2952.

[20] M. Xu, T. Liang, M. Shi, H. Chen, Chem. Rev. 113 (2013) 3766.

[21] Z. Sun, H. Chang, ACS Nano 8 (2014) 4133.

[22] Q. H. Wang, K. Kalantar-Zadeh, A. Kis, J. N. Coleman, M. S. Strano, Nat. Nanotechnol. 7 (2012) 699.

[23] H. Zeng, B. Zhu, K. Liu, J. Fan, X. Cui, Q. M. Zhang, Phys. Rev. B 86 (2012) 241301.

[24] B. Radisavljevic, A. Radenovic, J. Brivio, V. Giacometti, A. Kis, Nat. Nanotechnol. 6 (2011) 147.

[25] J. Qiao, X. Kong, Z.-X. Hu, F. Yang, W. Ji, Nat. Commun. 5 (2014) 4475.

[26] A. S. Rodin, A. Carvalho, A. H. Castro Neto, Phys. Rev. Lett. 112 (2014) 176801.

[27] S. P. Koenig, R. A. Doganov, H. Schmidt, A. H. Castro Neto, B. Oezyilmaz, Appl. Phys. Lett. 104 (2014) 103106.

[28] A. Favron, E. Gaufrès, F. Fossard, A. -L. Phaneuf-L’Heureux, N. Y-W. Tang, P. L. Lévesque, A. 
Loiseau, R. Leonelli, S. Francoeur, R. Martel, Nat. Mater. 14 (2015) 826.

[29] A. N. Rudenko, M. I. Katsnelson, Phys. Rev. B 89 (2014) 201408.

[30] C. Q. Han, M. Y. Yao, X. X. Bai, Lin Miao, F. F. Zhu, D. D. Guan, S. Wang, C. L. Gao, C. H. Liu, D. Qian, Y. Liu, J. F. Jia, Phys. Rev. B 90 (2014) 085101.

[31] T. Vy, R. Soklaski, Y. Liang, L. Yang, Phys. Rev. B 89 (2014) 235319.

[32] L. Liang, J. Wang, W. Lin, B. G. Sumpter, V. Meunier, M. Pan, Nano Lett. 14, 6400 (2014).

[33] Q. Wei, X. Peng, Appl. Phys. Lett. 104 (2014) 251915.

[34] G. Qin, Q.-B. Yan, Z. Qin, S.-Y. Yue, H.-J. Cui, Q.-R. Zheng, G. Su, Sci. Rep. 4 (2014) 6946.

[35] J.-W. Jiang, H. S. Park, Nat. Commun. 5 (2014) 4727.

[36] R. Fei, L. Yang, Nano Lett. 14 (2014) 2884.

[37] R. Fei, A. Faghaninia, R. Soklaski, J.-A. Yan, C. Lo, L. Yang, Nano Lett. 14 (2014) 6393.

[38] X. M. Wang, A. M. Jones, K. L. Seyler, V. Tran, Y. C. Jia, H. Zhao, H. Wang, L. Yang, X. D. Xu, F. N. Xia, Nat. Nanotechnol. 10 (2015) 517.

[39] L. Li, Y. Yu, G. J. Ye, Q. Ge, X. Ou, H. Wu, D. Feng, X. H. Chen, Y. Zhang, Nat. Nanotechnol. 9 (2014) 372.

[40] M. Buscema, D. J. Groenendijk, G. A. Steele, H. S. J. van der Zant, A. Castellanos-Gomez, Nat. Commun. 5 (2014) 4651.

[41] M. Buscema, D. J. Groenendijk, S. I. Blanter, G. A. Steele, H. S. J. van der Zant, A. Castellanos-Gomez, Nano Lett. 14 (2014) 3347.

[42] F. Xia, H. Wang, Y. Jia, Nat. Commun. 5 (2014) 4458.

[43] J. O. Island, G. A. Steele, H. S. J. van der Zant, A. Castellanos-Gomez, 2D Mater. 2 (2015) 011002 .

[44] L. Kou, C. Chen, S. C. Smith, J. Phys. Chem. Lett. 6 (2015) 2794.

[45] A. C. Ferrari, D. M. Basko, Nat. Nanotechnol. 8 (2013) 235.

[46] J.-H. Fan, P. Gao, A.-M. Zhang, B.-R. Zhu, H.-L. Zeng, X.-D. Cui, R. He, Q.-M. Zhang, J. Appl. Phys. 115 (2014) 053527.

[47] K. K. Mani, R. Ramani, Phys. Status Solidi B 61 (1974) 659.

[48] R. J. Nemanich, G. Lucovsky, S. A. Solin, Solid State Commun. 23 (1977) 117.

[49] L. M. Malard, M. H. D. Guimaraes, D. L. Mafra, M. S. C. Mazzoni, A. Jorio, Phys. Rev. B 79 (2009) 125426.

[50] S. K. Saha, U. V. Waghmare, H. R. Krishnamurthy, A. K. Sood, Phys. Rev. B 78 (2008) 165421.

[51] K. H. Michel, B. Verberck, Phys. Rev. B 85 (2012) 094303.

[52] P. H. Tan, W. P. Han, W. J. Zhao, Z. H. Wu, K. Chang, H. Wang, Y. F. Wang, N. Bonini, N. Marzari, N. Pugno, G. Savini, A. Lombardo, A. C. Ferrari, Nat. Mater. 11 (2012) 294.

[53] L. M. Malarda, M. A. Pimentaa, G. Dresselhausb, M. S. Dresselhaus, Phys. Rep. 473 (2009) 51.

[54] S. Dong, A. M. Zhang, K. Liu, J. T. Ji, Y. G. Ye, X. G. Luo, X. H. Chen, X. L. Ma, Y. H. Jie, C. F. Chen, X. Q. Wang, Q. M. Zhang, arXiv preprint:1503.06577.

[55] X. Luo, X. Lu, G. K. W. Koon, A. H. C. Neto, B. Oezyilmaz, Q. Xiong, S. Y. Quek, Nano Lett. 15 (2015) 3931.

[56] J. L. Verble, T. J. Wietling, P. R. Reed, Solid State Commun. 11 (1972) 941.

[57] X. Luo, X. Lu, C. Cong, T. Yu, Q. Xiong, S. Y. Quek, Sci. Rep. 5 (2015) 14565.

[58] C. H. Lui, Z. Ye, C. Keiser, E. B. Barros, R. He, Appl. Phys. Lett. 106 (2015) 041904.

[59] X. Lu, M. I. BaktiUtama, J. H. Lin, X. Luo, Y. Y. Zhao, J. Zhang, S. T. Pantelides, W. Zhou, S. Y. Quek, Q. H. Xiong, Adv. Mater. 27 (2015) 4502. 
[60] C. H. Lui, Z. P. Ye, C. Ji, K. -C. Chiu, C. -T. Chou, T. I. Andersen, C. Means-Shively, H. Anderson, J. -M. Wu, T. Kidd, Y. -H. Lee, Rui He, Phys. Rev. B 91 (2015) 165403.

[61] N. S. Luo, P. Ruggerone, J. P. Toennies, Phys. Rev. B 54 (1996) 5051.

[62] C. H. Lui, T. F. Heinz, Phys. Rev. B 87 (2013) 121404.

[63] C. H. Lui, L. M. Malard, S. Kim, G. Lantz, F. E. Laverge, R. Saito, T. F. Heinz, Nano Lett. 12 (2012) 5539.

[64] X. Zhang, W. P. Han, J. B. Wu, S. Milana, Y. Lu, Q. Q. Li, A. C. Ferrari, P. H. Tan, Phys. Rev. B 87 (2013) 115413.

[65] M. Boukhicha, M. Calandra, M.-A. Measson, O. Lancry, A. Shukla, Phys. Rev. B 87 (2013) 195316.

[66] Y. Zhao, X. Luo, H. Li, J. Zhang, P. T. Araujo, C. K. Gan, J. Wu, H. Zhang, S. Y. Quek, M. S. Dresselhaus, Q. Xiong, Nano Lett. 13 (2013) 1007.

[67] G. Plechinger, S. Heydrich, J. Eroms, D. Weiss, C. Schueller, T. Korn, Appl. Phys. Lett. 101 (2012) 101906.

[68] M. O'Brien, N. McEvoy, D. Hanlon, K. Lee, R. Gatensby, J. N. Coleman, G. S. Duesberg, Phys. Status Solidi B 252 (2015) 2385.

[69] X. Xi, L. Zhao, Z. Wang, H. Berger, L. Forro, J. Shan, K. F. Mak, Nat. Nanotechnol. 10 (2015) 765.

[70] G. Froehlicher, E. Lorchat, F. Fernique, C. Joshi, A. Molina-Sanchez, L. Wirtz, S. Berciaud, Nano Lett. 15 (2015) 6481.

[71] X. Ling, L. Liang, S. Huang, A. A. Puretzky, D. B. Geohegan, B. G. Sumpter, J. Kong, V. Meunier, M. S. Dresselhaus, Nano Lett. 15 (2015) 4080.

[72] A. Bosak, M. Krisch, M. Mohr, J. Maultzsch, C. Thomsen, Phys. Rev. B 75 (2007) 153408.

[73] R. Ganatra, Q. Zhang, ACS Nano 8 (2014) 4074.

[74] A. Molina-Sanchez, L. Wirtz, Phys. Rev. B 84 (2011) 155413.

[75] S. J. Sandoval, D. Yang, R. F. Frindt, J. C. Irwin, Phys. Rev. B 44 (1991) 3955.

[76] Y. Cai, J. Lan, G. Zhang, Y.-W. Zhang, Phys. Rev. B 89 (2014) 035438.

[77] C. Lee, H. Yan, L. E. Brus, T. F. Heinz, J. Hone, S. Ryu, ACS Nano 4 (2010) 2695.

[78] T. J. Wieting, Solid State Commun. 12 (1973) 931.

[79] G. Plechinger, P. Nagler, J. Kraus, N. Paradiso, C. Strunk, C. Schueller, T. Korn, Phys. Status Solidi-RRL 9 (2015) 457.

[80] R. He, J.-A. Yan, Z. Yin, Z. Ye, G. Ye, J. Cheng, J. Li, C. H. Lui, Nano Lett. (2016)

[81] S. Sugai, I. Shirotani, Solid State Commun. 53 (1985) 753.

[82] J. Ribeiro-Soares, R. M. Almeida, L. G. C. Ado, M. S. Dresselhaus, A. Jorio, Phys. Rev. B 91 (2015) 205421.

[83] S. A. Safron, G. G. Bishop, J. Duan, E. S. Gillman, J. G. Skofronick, N. S. Luo, P. Ruggerone, J. Phys. Chem. 97 (1993) 2270.

[84] Z. X. Hu, X. H. Kong, J. S. Qiao, B. Normand, W. Ji, arXiv preprint:1503.06735 (2015).

[85] H. Zhang, C.-X. Liu, X.-L. Qi, X. Dai, Z. Fang, S.-C. Zhang, Nat. Phys. 5 (2009) 438.

[86] Y. Y. Zhao, X. Luo, J. Zhang, J. X. Wu, X. X. Bai, M. X. Wang, J. F. Jia, H. L. Peng, Z. F. Liu, S. Y. Quek, Q. H. Xiong, Phys. Rev. B 90 (2014) 245428.

[87] M. F. Craciun, S. Russo, M. Yamamoto, J. B. Oostinga, A. F. Morpurgo, S. Tarucha, Nat. Nanotechnol. 4 (2009) 383.

[88] K. Zou, F. Zhang, C. Capp, A. H. MacDonald, J. Zhu, Nano Lett. 13 (2013) 369. 
[89] C. H. Lui, Z. Li, K. F. Mak, E. Cappelluti, T. F. Heinz, Nat. Phys. 7 (2011) 944.

[90] T. Taychatanapat, K. Watanabe, T. Taniguchi, P. Jarillo-Herrero, Nat. Phys. 7 (2011) 621.

[91] W. Bao, L. Jing, J. Velasco Jr, Y. Lee, G. Liu, D. Tran, B. Standley, M. Aykol, S. B. Cronin, D. Smirnov, M. Koshino, E. McCann, M. Bockrath, C. N. Lau, Nat. Phys. 7 (2011) 948.

[92] L. Zhang, Y. Zhang, J. Camacho, M. Khodas, I. Zaliznyak, Nat. Phys. 7 (2011) 953.

[93] J.-B. Wu, Z. -X. Hu, X. Zhang, W. -P. Han, Y. Lu, W. Shi, X. -F. Qiao, M. Ijiäs, S. Milana, W. Ji, A. C. Ferrari, P. -H. Tan, ACS Nano 9 (2015) 7440.

[94] A. K. Geim, I. V. Grigorieva, Nature 499 (2013) 419.

[95] X. P. Hong, J. Kim, S. -F. Shi, Y. Zhang, C. H. Jin, Y. H. Sun, S. Tongay, J. Q. Wu, Y. F. Zhang, F. Wang, Nat. Nanotechnol. 9 (2014) 682.

[96] C. -H. Lee, G. -H. Lee, A. M. van der Zande, W. Chen, Y. L. Li, M. Han, X. Cui, G. Arefe, C. Nuckolls, T. F. Heinz, J. Guo, J. Hone, P. Kim, Nat. Nanotechnol. 9 (2014) 676.

[97] L. Britnell, R. V. Gorbachev, R. Jalil, B. D. Belle, F. Schedin, A. Mishchenko, T. Georgiou, M. I. Katsnelson, L. Eaves, S. V. Morozov, N. M. R. Peres, J. Leist, A. K. Geim, K. S. Novoselov, L. A. Ponomarenko, Science 335 (2012) 947.

[98] G. W. Shim, K. Yoo, S.-B. Seo, J. Shin, D. Y. Jung, I.-S. Kang, C. W. Ahn, B. J. Cho, S.-Y. Choi, ACS Nano 8 (2014) 6655.

[99] H. Fang, C. Battaglia, C. Carraro, S. Nemsak, B. Ozdol, J. S. Kang, H. A. Bechtel, S. B. Desai, F. Kronast, A. A. Unal, G. Conti, C. Conlon, Palsson, K. Gunnar, M. C. Martin, A. M. Minor, C. S. Fadley, E. Yablonovitch, R. Maboudian, A. Javey, PNAS 111 (2014) 6198.

[100] M. V. Kamalakar, A. Dankert, J. Bergsten, T. Ive, S. P. Dash, Appl. Phys. Lett. 105 (2014) 212405.

[101] S. Latini, T. Olsen, K. S. Thygesen, arXiv preprint:1509.07972 (2015).

[102] E. V. Calman, C. J. Dorow, M. M. Fogler, L. V. Butov, S. Hu, A. Mishchenko, A. K. Geim, arXiv preprint:1510.04410(2015). 


\section{Figure captions}

Fig. 1. (a), (c) and (e) are the side views of graphene, $\mathrm{MoS}_{2}$ and black phosphorus lattice structures. And (b), (d) and (f) are the corresponding top views, respectively.

Fig. 2. (a) DFT calculations of the symmetry and frequencies of $\mathrm{C}$ mode. (b) Frequency evolution of $\mathrm{C}$ mode with the number of layers. (c) Linear chain fitting of $\mathrm{C}$ mode [52].

Fig. 3. (a) 2ZO' Raman spectra of 2- to 20-layer graphene and graphite. (b) Frequency evolution of compression mode ZO' obtained from (a) and fitting results using linear chain model. (c) Electronic scattering processes for double phonon resonance Raman of 2ZO' mode in bi-layer graphene [62].

Fig. 4. (a) Vibration patterns of Raman-active modes in $\mathrm{MoS}_{2}$. (b) Shear and compression modes in the Raman spectra of few-layer $\mathrm{MoS}_{2}$. (c) \& (d) Fitting results for shear and compression modes using linear chain model, respectively [23].

Fig. 5. (a) Shear modes in few-layer $\mathrm{MoSe}_{2}$ under parallel polarization configutration (xx) [59]. (b) Evolution of low-frequency interlayer modes with thickness in few-layer $\mathrm{WSe}_{2}$ [66]. (c) Low-frequency Raman spectra of $\mathrm{MoTe}_{2}$ from monolayer to bulk [70]. (d) Low-frequency Raman modes of 2- to 10-layer $\operatorname{ReS}_{2}$ [80].

Fig. 6. (a) Low-frequency Raman spectra of multilayer and bulk black phosphorus. (b) and (c) are frequency dependence of C and CCM modes on layer number and the corresponding linear chain fitting. (d) and (e) are the vibration patterns of the modes. (f) \&(g) Evolution of low-frequency Raman modes with polarization of incident light [54].

Fig. 7. (a)\&(b) Low-frequency Raman spectra of multilayer and bulk $\mathrm{Bi}_{2} \mathrm{Se}_{3}$ and $\mathrm{Bi}_{2} \mathrm{Te}_{3}$ under parallel polarization (xx) configuration, respectively. (c)\&(d) Linear chain model with and without a substrate [86].

Fig. 8. (a) Lattice structure and symmetry of trilayer graphene with ABA and ABC stacking and vibration patterns of interlayer modes in trilayer graphene. (b) Phonon dispersions of low frequency modes in trilayer graphene with ABA and ABC stacking. (c) Low frequency Raman spectra for the two kinds of stacking methods [58].

Fig. 9. Shear and compression modes observed in the Stokes and anti-Stokes Raman 
spectra of twisted multilayer graphene [93]. 
a

:? : : :

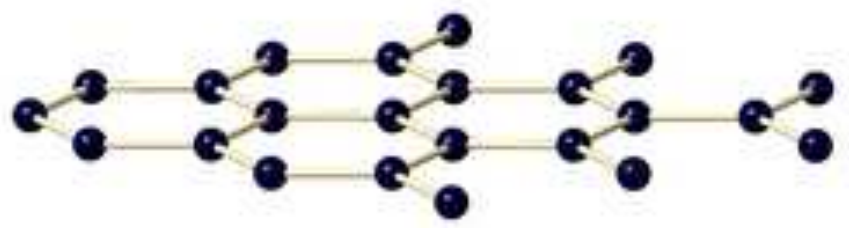

b

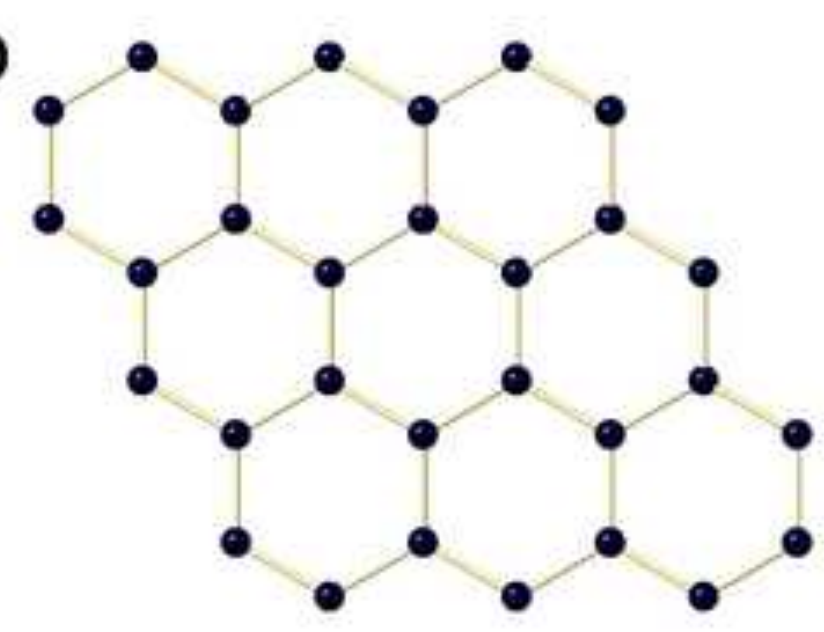

C
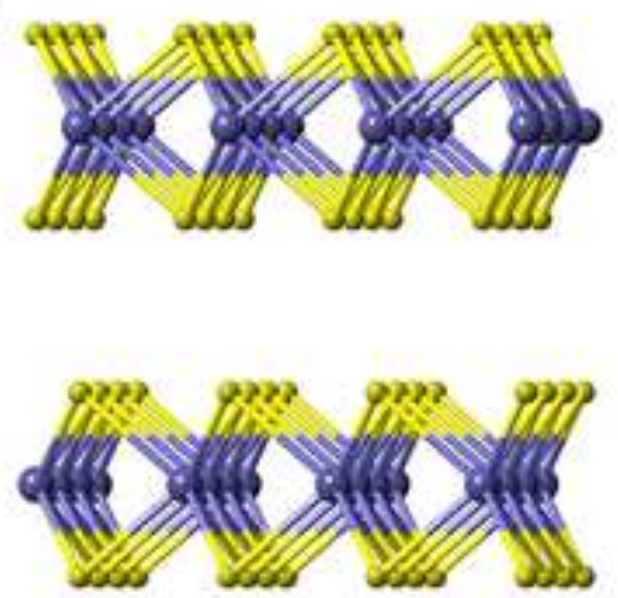

d

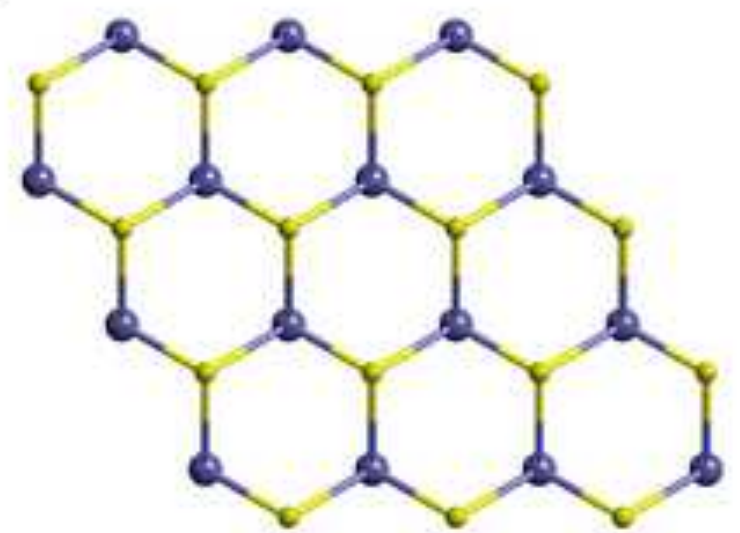

e
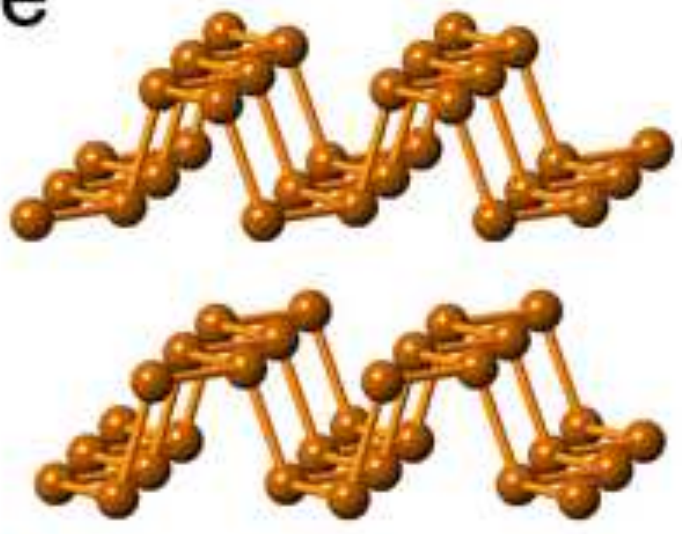

f

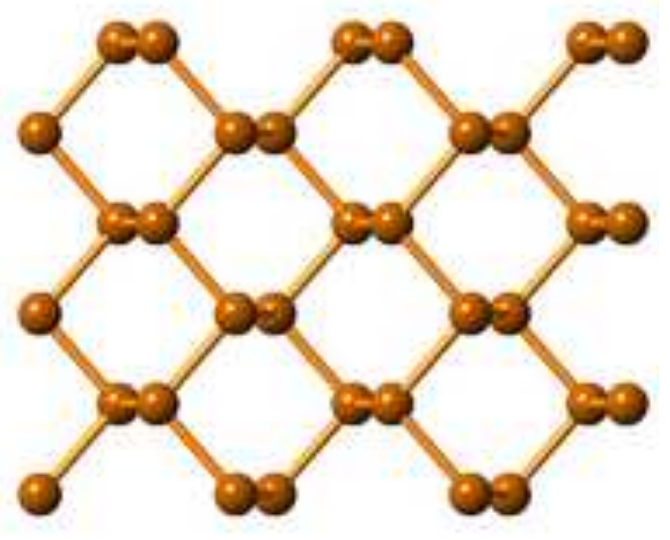



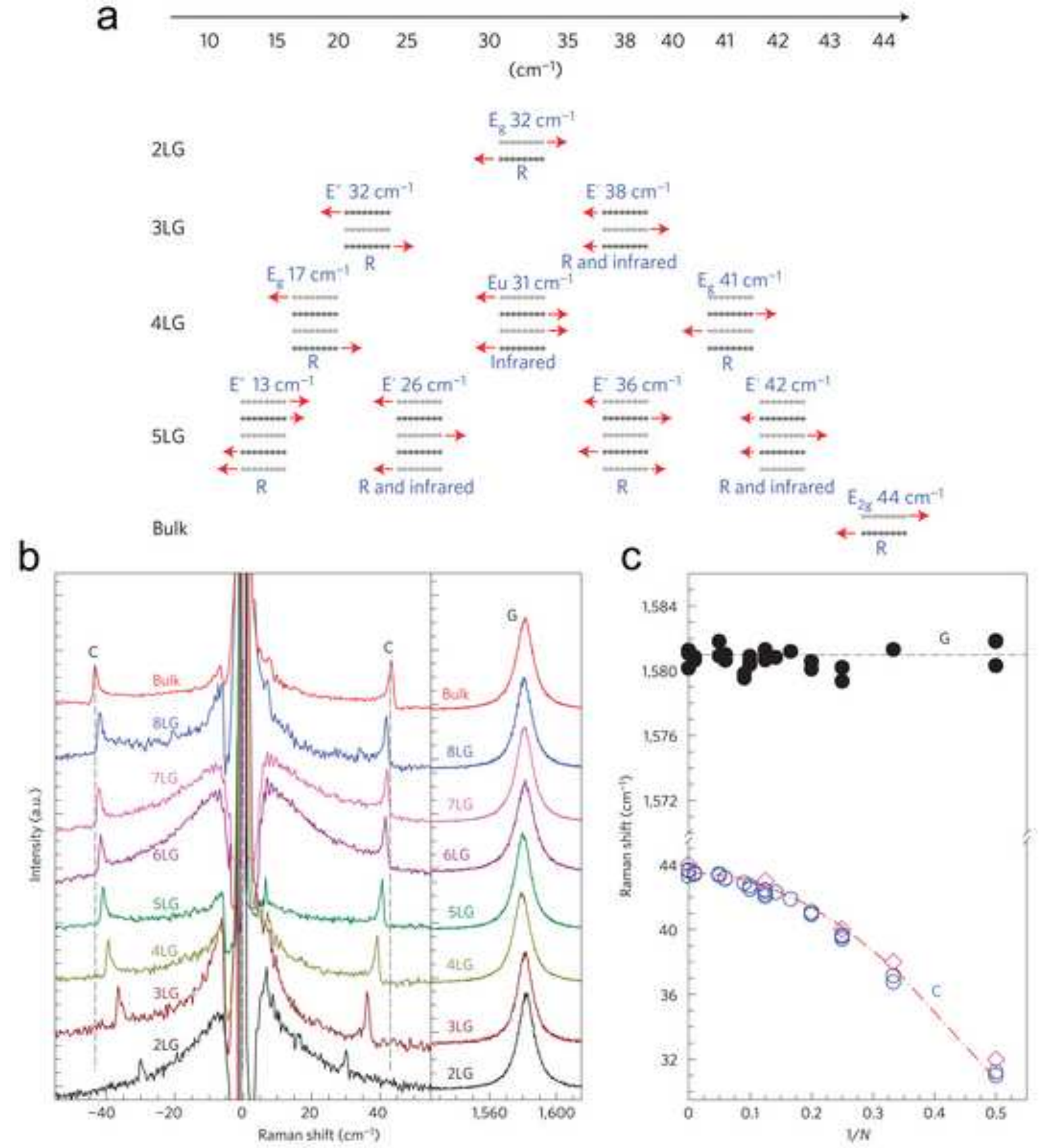
a

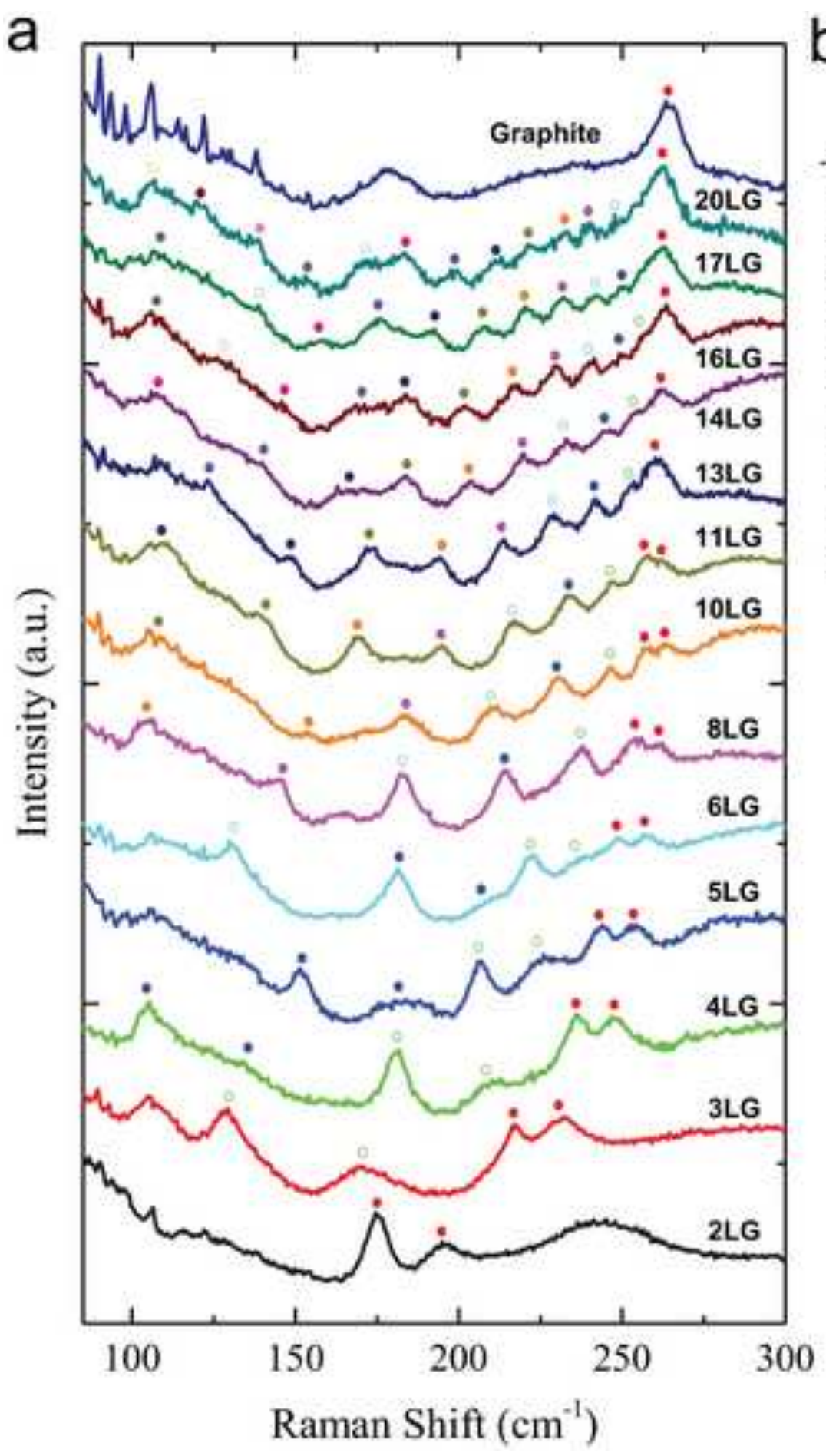

b

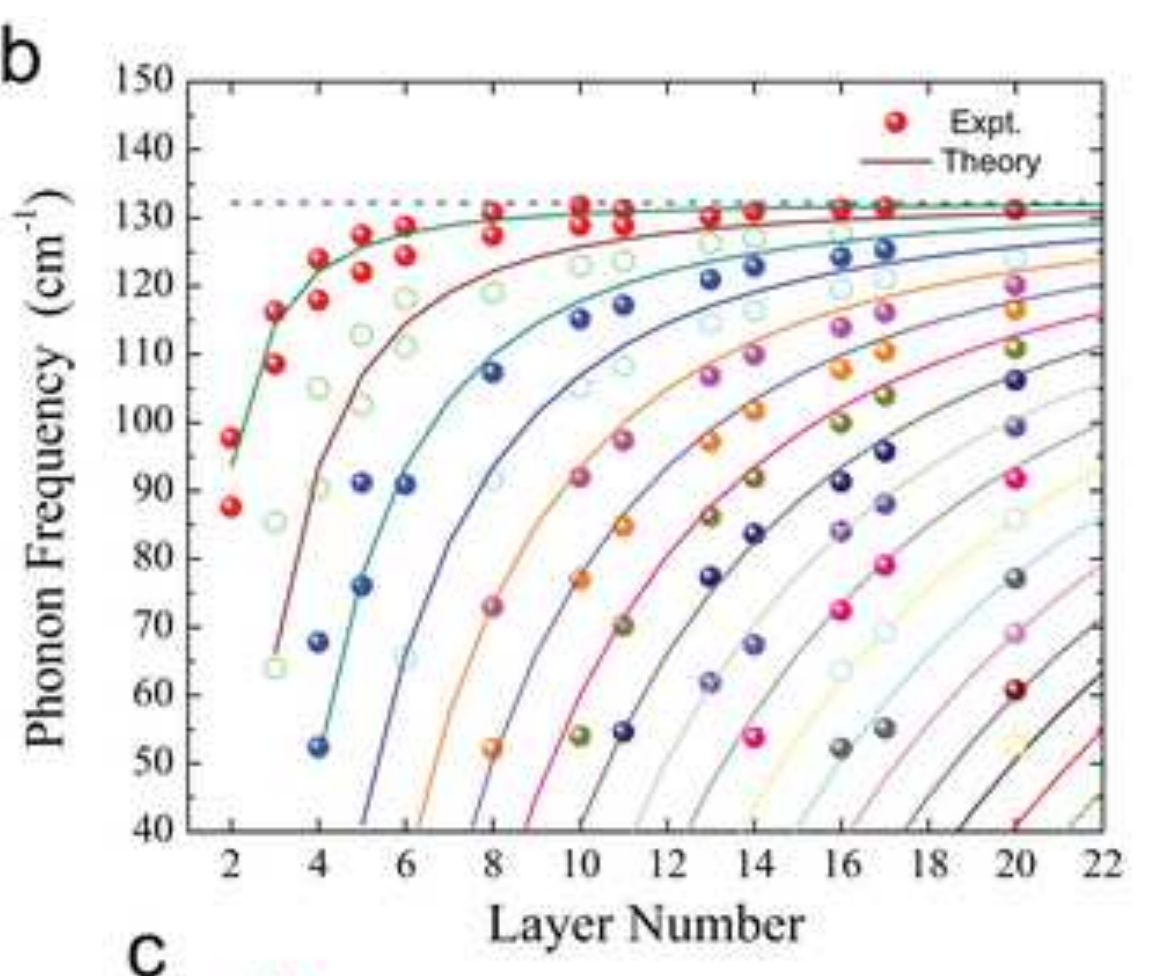

C

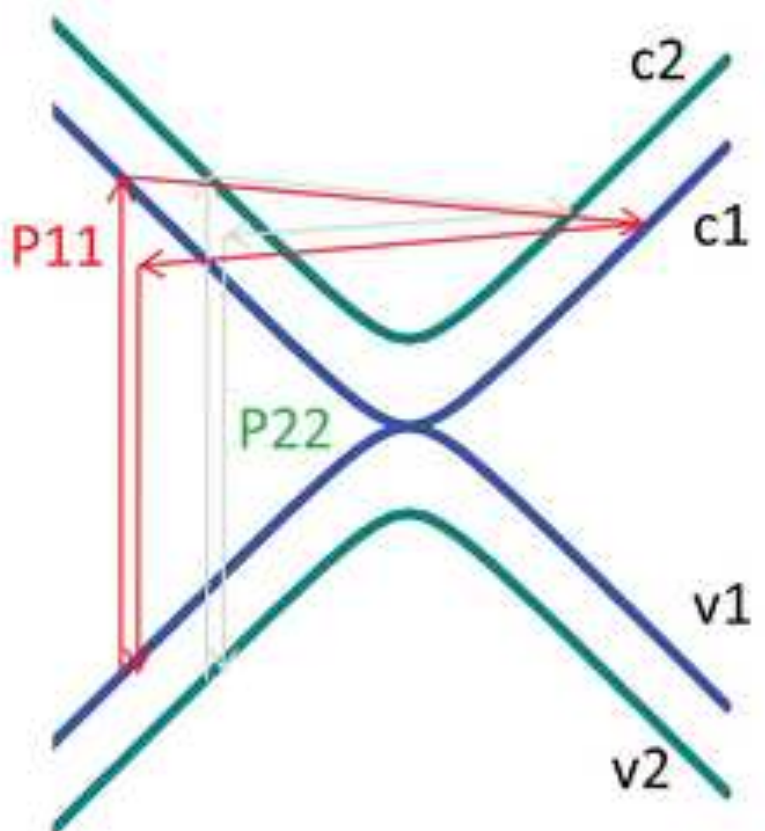

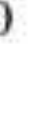


a
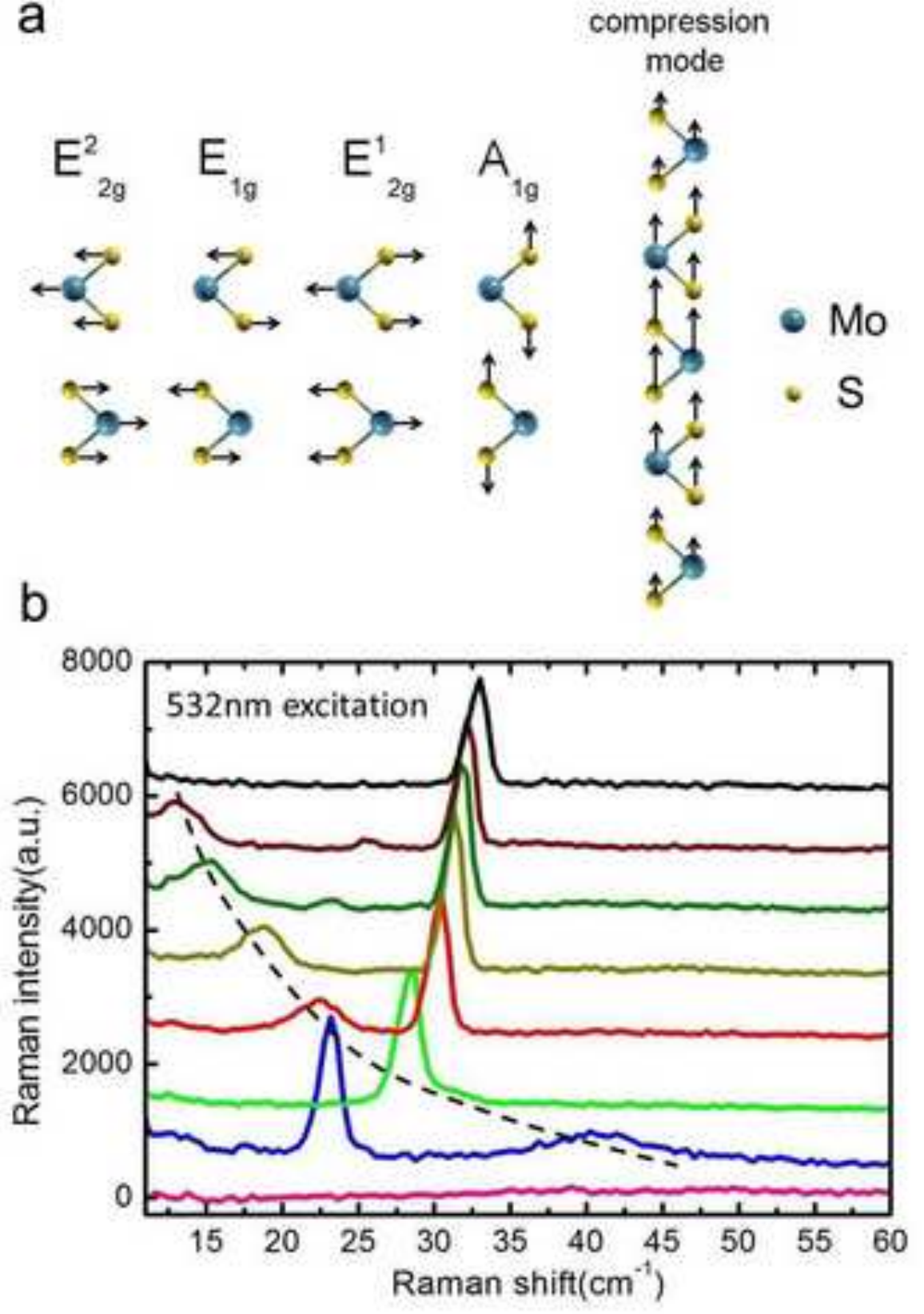

C

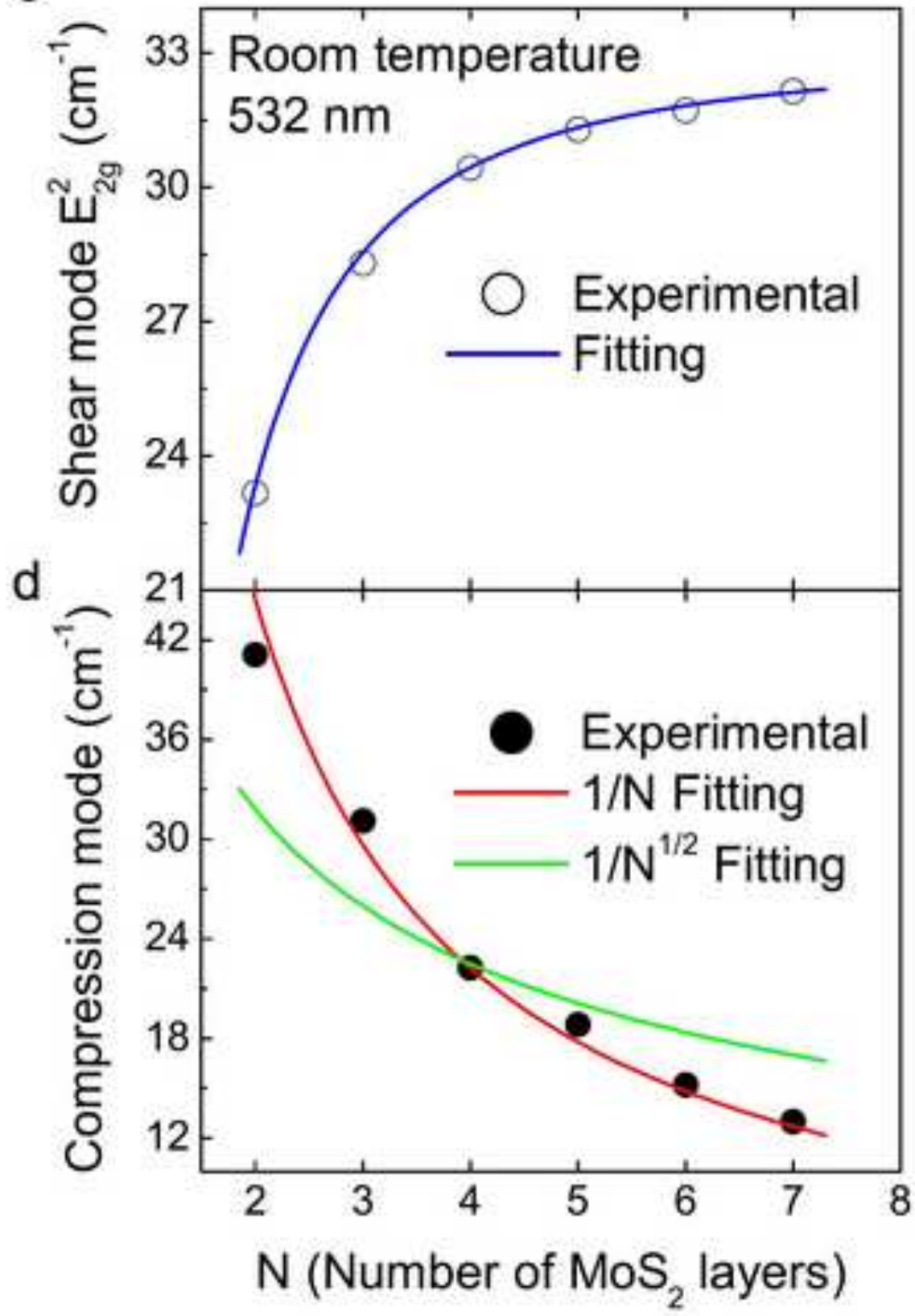




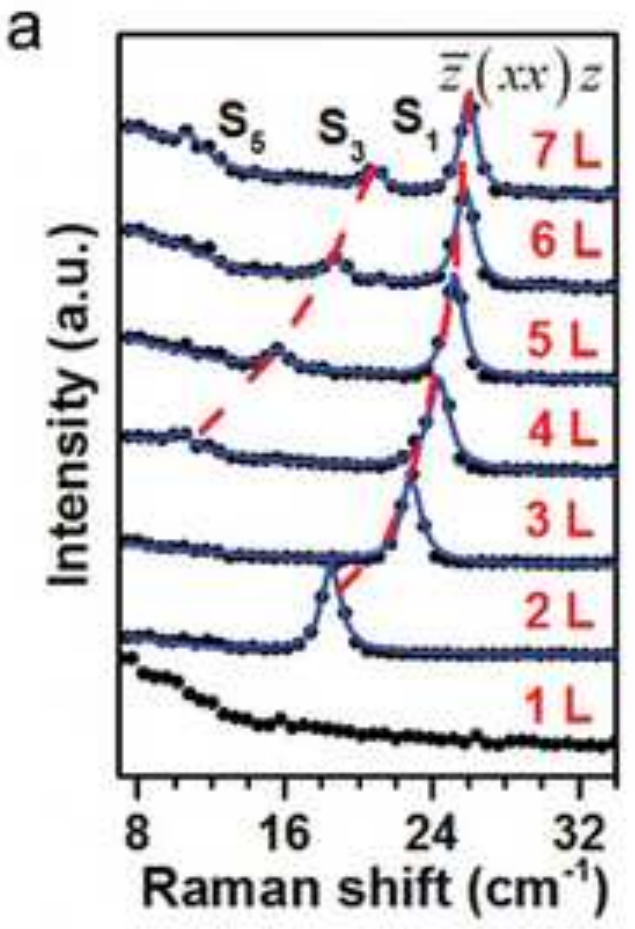

b

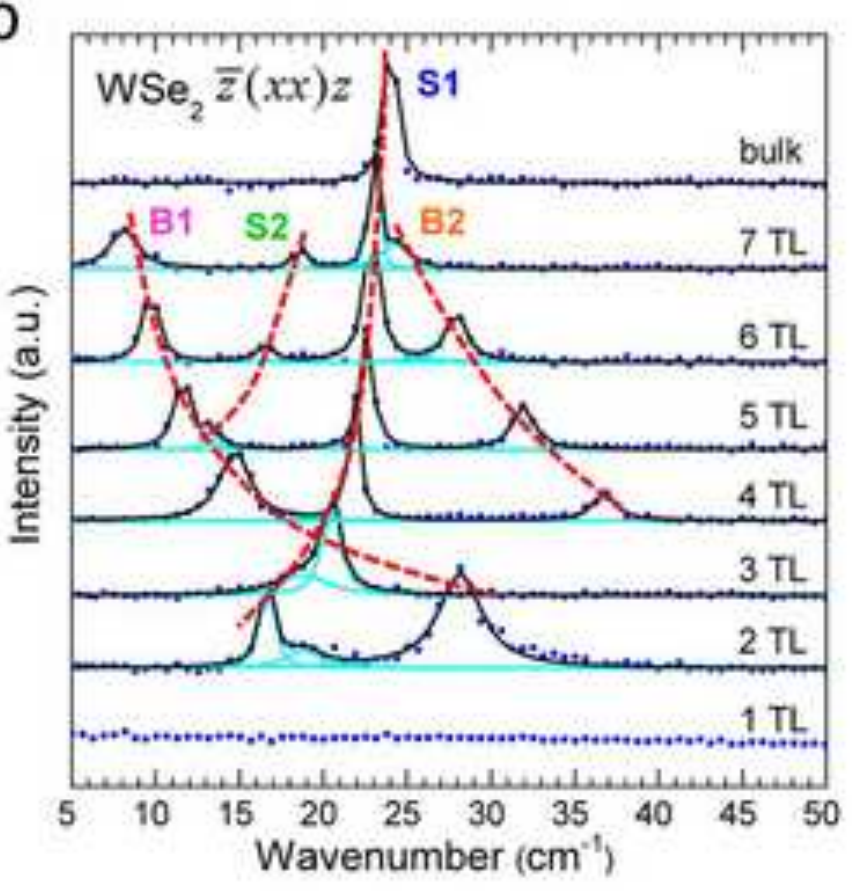

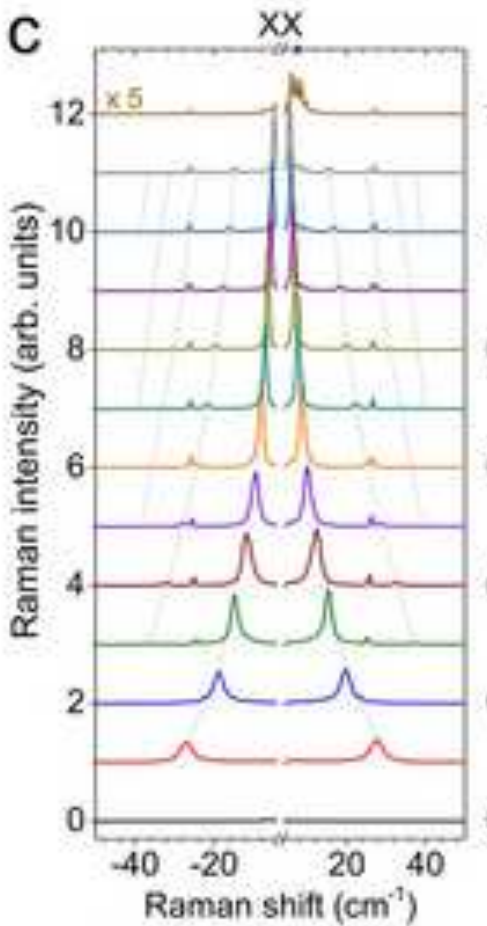

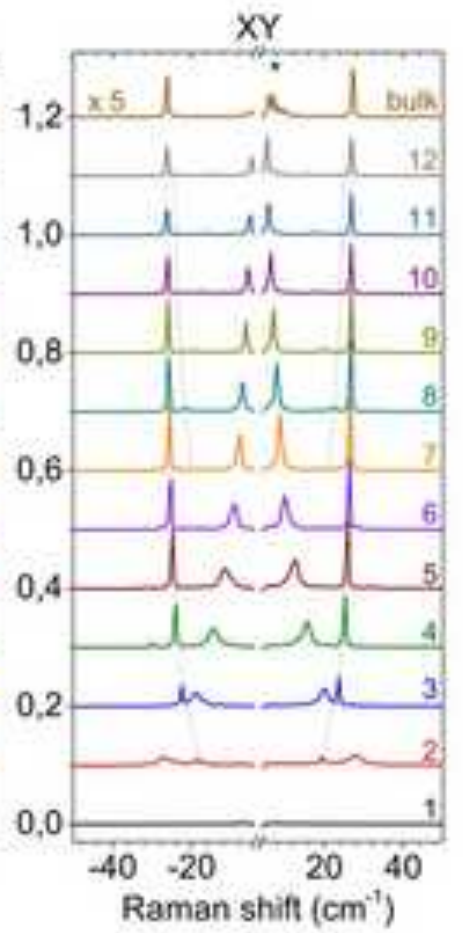

d

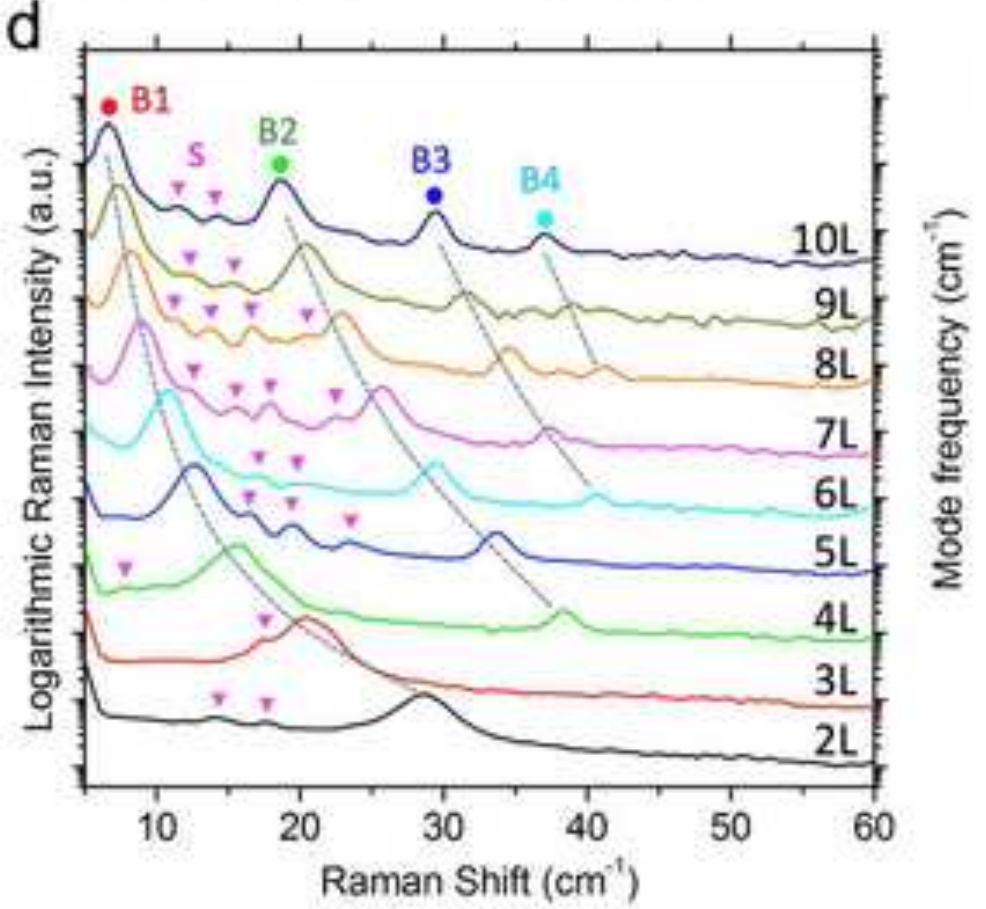



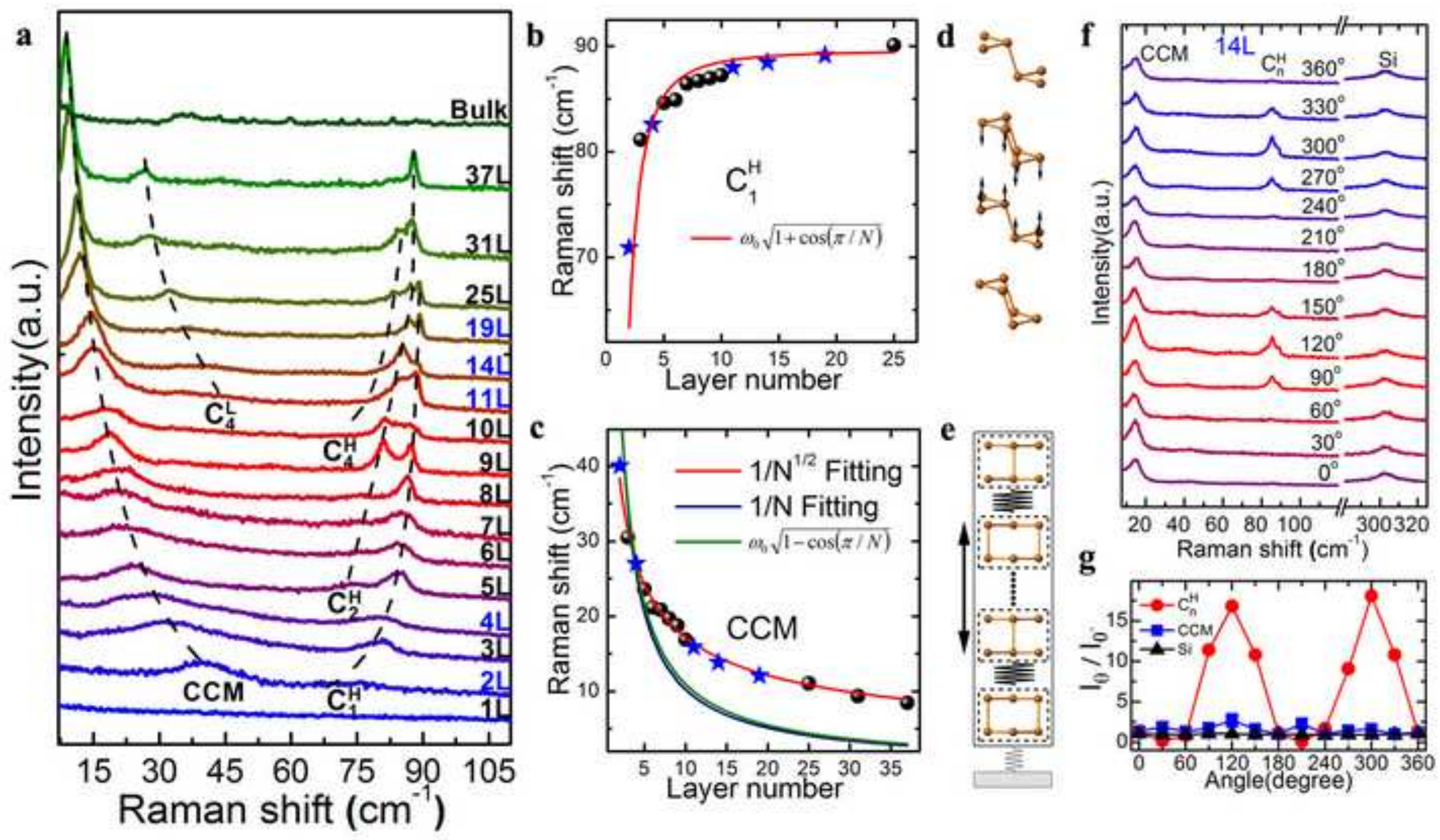

g

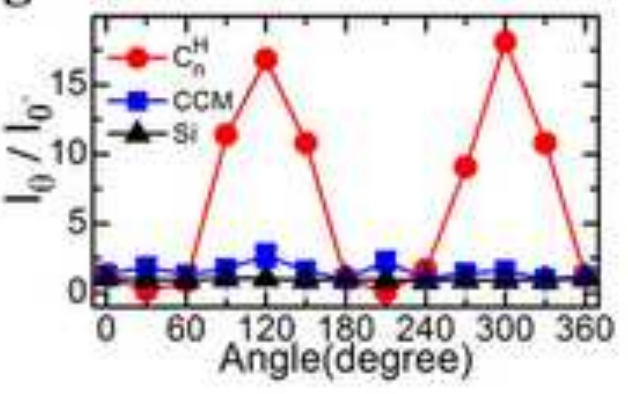

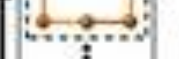

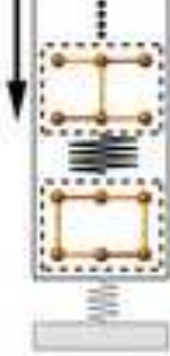


a

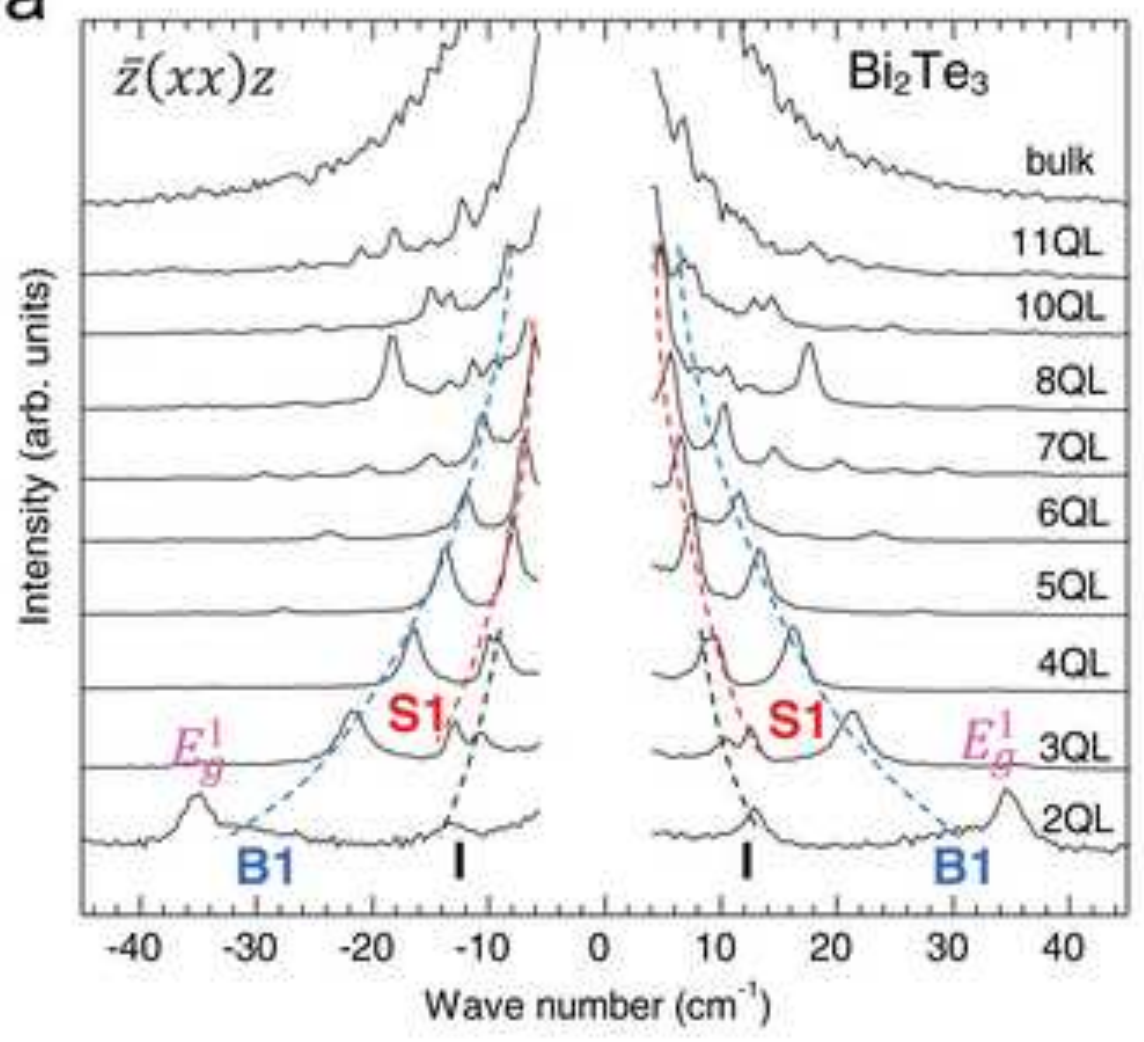

C

Free Linear Chain Model

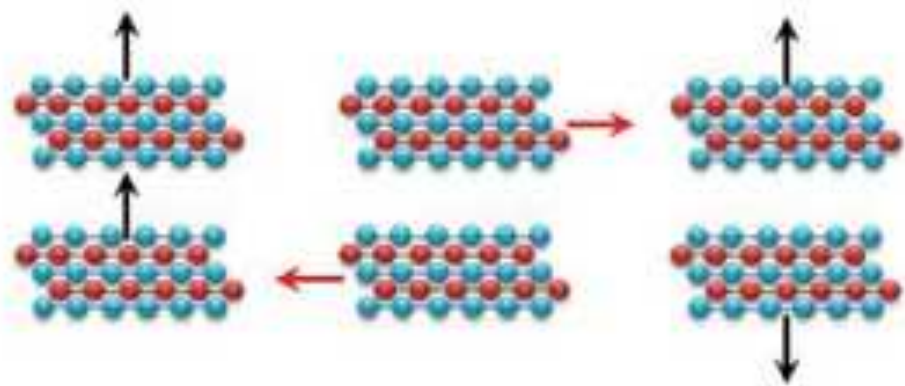

Acoustic Mode S1

B1 b

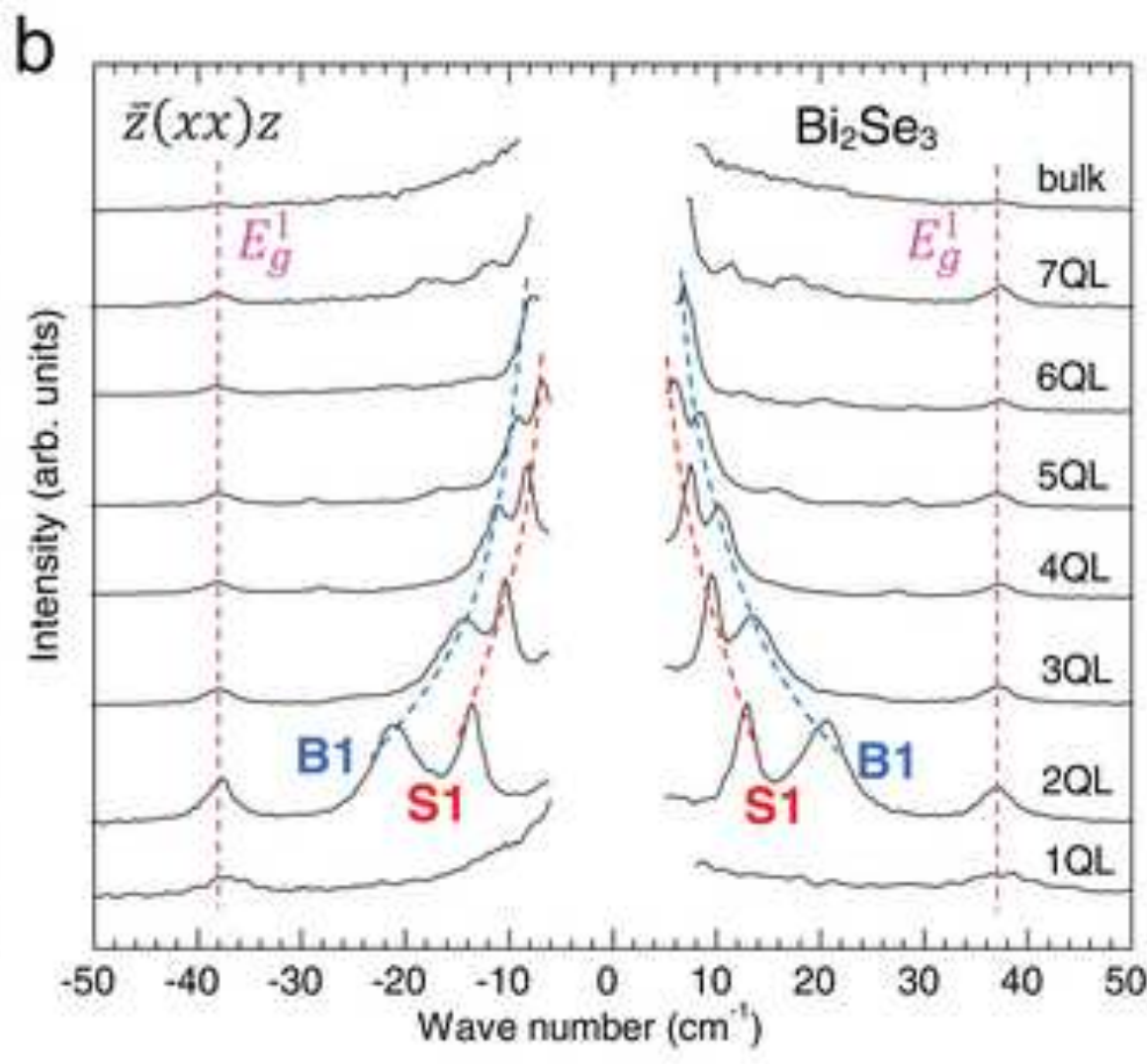

d Linear Chain Modelwith a Substrate

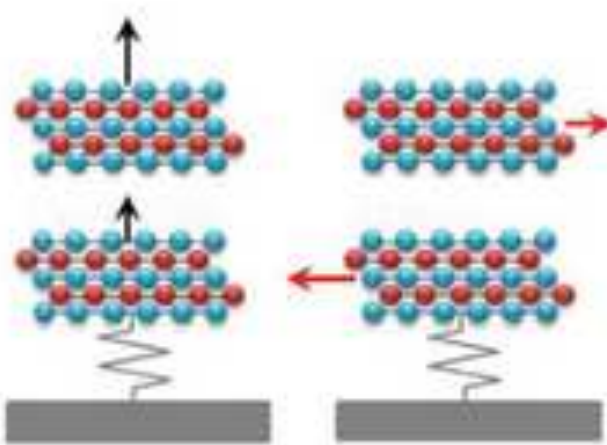

Interface Mode S1

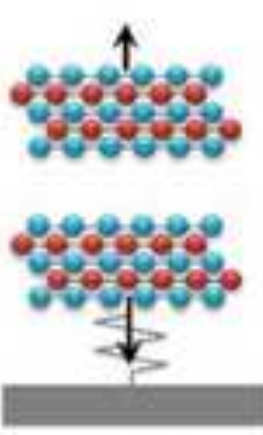

B1
B1 
a $\quad$ ABA trilayer $\left(D_{3 h}\right)$

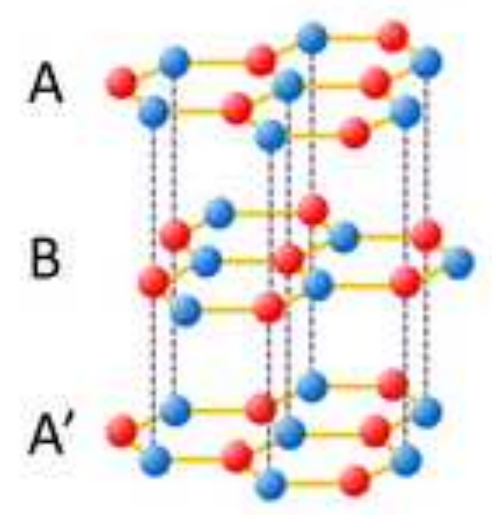

Low shear mode $22 \mathrm{~cm}^{-1}$

000อ० $\Rightarrow$

o 000

\&oopoo

$A B A: E^{\prime \prime}$, Raman

$A B C$ : $E_{g}$, Raman
High shear mode $37 \mathrm{~cm}^{-1}$

-

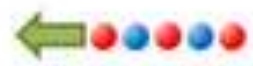

- 000 को

$E^{\prime}$, Raman + IR

$E_{i,}$ IR
ABC trilayer $\left(D_{3 d}\right)$

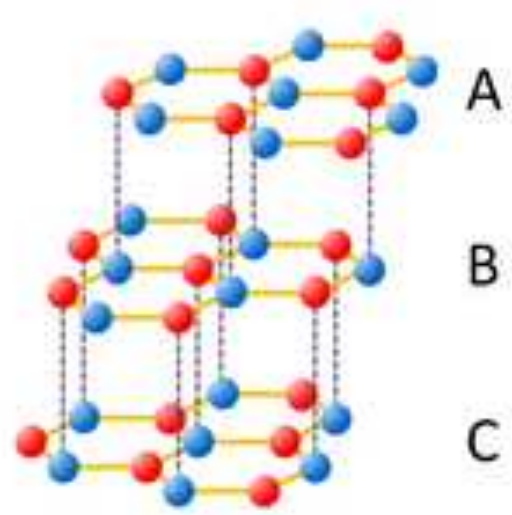

C

b

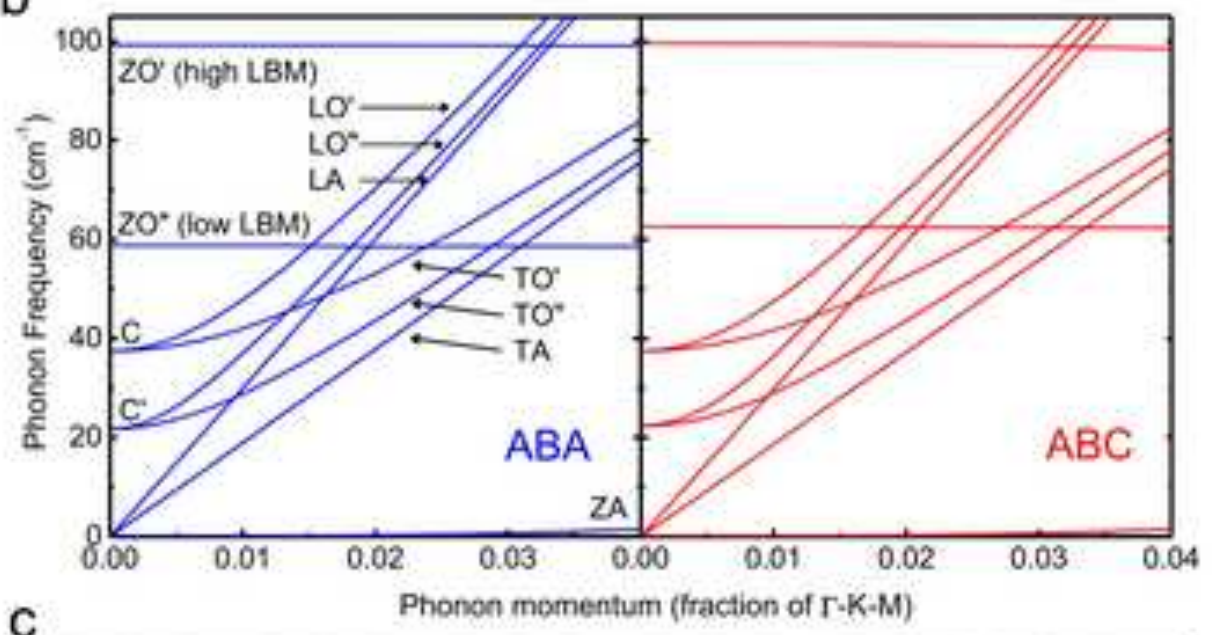

C

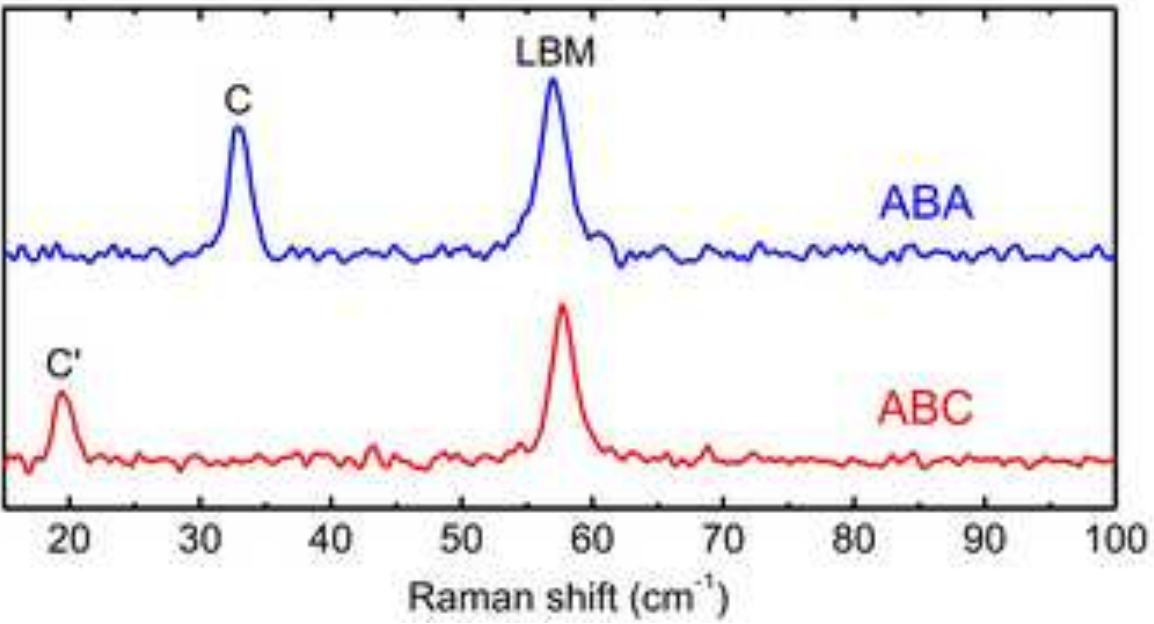




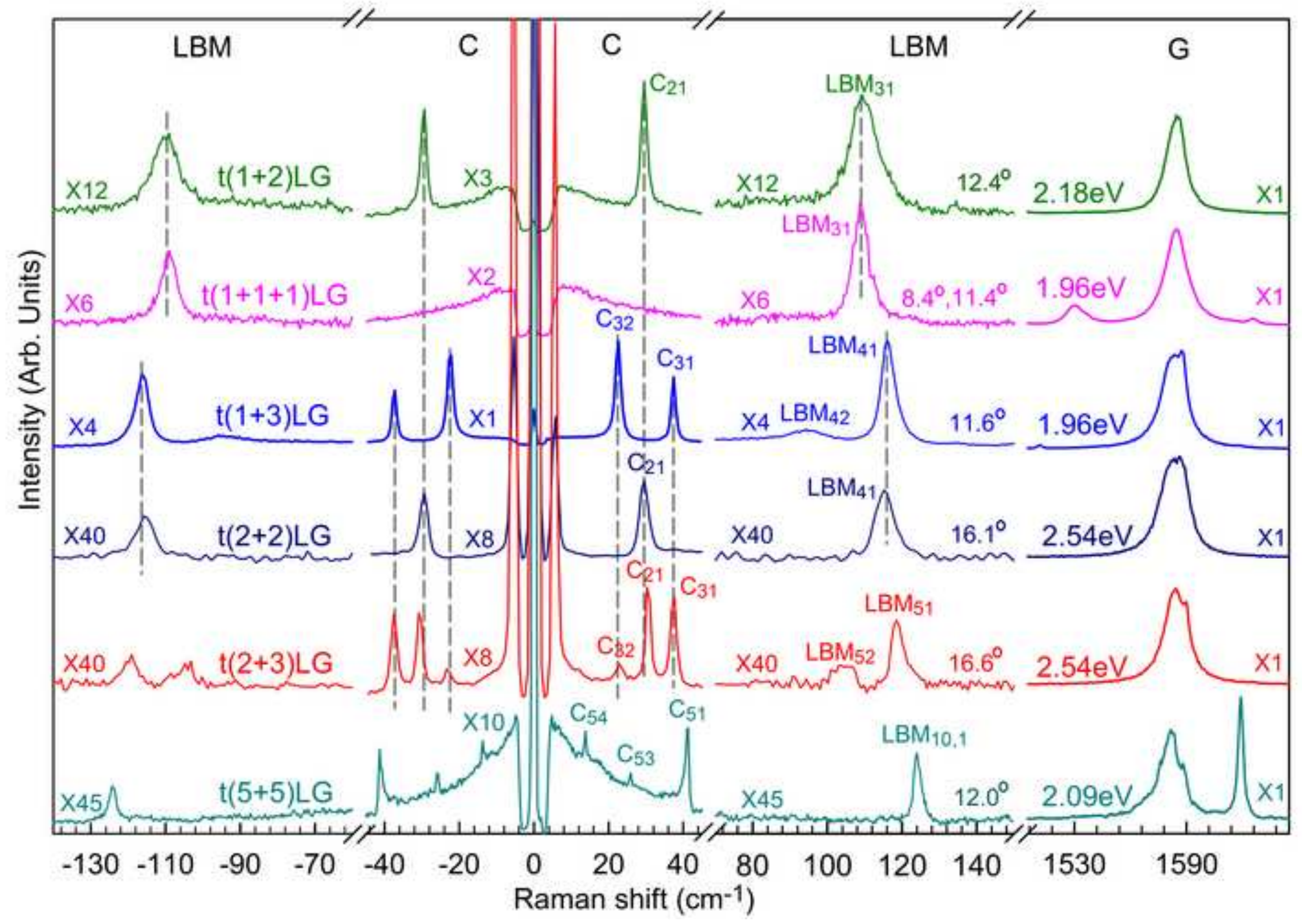

